\title{
Consistency measures of linguistic preference relations with hedges
}

DOI:

10.1109/TFUZZ.2018.2856107

\section{Document Version}

Accepted author manuscript

Link to publication record in Manchester Research Explorer

\section{Citation for published version (APA):}

Wang, H., Xu, Z. S., Zeng, X., \& Liao, H. (2018). Consistency measures of linguistic preference relations with hedges. IEEE Transactions on Fuzzy Systems. https://doi.org/10.1109/TFUZZ.2018.2856107

\section{Published in:}

IEEE Transactions on Fuzzy Systems

\section{Citing this paper}

Please note that where the full-text provided on Manchester Research Explorer is the Author Accepted Manuscript or Proof version this may differ from the final Published version. If citing, it is advised that you check and use the publisher's definitive version.

\section{General rights}

Copyright and moral rights for the publications made accessible in the Research Explorer are retained by the authors and/or other copyright owners and it is a condition of accessing publications that users recognise and abide by the legal requirements associated with these rights.

\section{Takedown policy}

If you believe that this document breaches copyright please refer to the University of Manchester's Takedown Procedures [http://man.ac.uk/04Y6Bo] or contact uml.scholarlycommunications@manchester.ac.uk providing relevant details, so we can investigate your claim.

\section{OPEN ACCESS}




\title{
Consistency measures of linguistic preference relations with hedges
}

\author{
Hai Wang, Zeshui Xu, Senior Member, IEEE, Xiao-Jun Zeng, Member, IEEE, and Huchang Liao, Senior \\ Member, IEEE
}

\begin{abstract}
Modeling linguistic information is vital for qualitative decision making (QDM). Compared with single linguistic terms, the complex linguistic expressions (CLEs) are more powerful and flexible to express linguistic opinions under uncertainties. Among the existing types of CLEs, the linguistic terms with weakened hedges (LTWHs), which focus on the uncertainty of using single terms, can be used to model the linguistic expressions in natural languages. This paper concentrates on the application of LTWHs in the framework of QDM with preference relations. The concept of linguistic preference relations with hedges (LHPRs) are presented after a new computational model of LTWHs is formed. Some consistency concepts, such as weak consistency and additive consistency, are then defined and their properties are studied. Especially, the theories and algorithms for consistency checking and improving are proposed. Finally, the availability of the proposed technique is demonstrated by a real application. Different from many studies related to consistency measures, we make use of fuzzy weighted digraphs to develop the theories and algorithms in a visible manner. Moreover, for consistency improving, the degree of consistency is measured by linguistic terms rather than numerical values so that the threshold of satisfactory consistency is interpretable.
\end{abstract}

Index Terms-Preference relations, Consistency measures, Linguistic terms with weakened hedges, Linguistic term sets, Transitivity.

\section{INTRODUCTION}

Qualitative information is generally inevitable and ubiquitous in practical process of collecting decision information. This is due to the nature of objects or the complexity of the focused problems [1]. To manipulate such kind of information, approaches for qualitative decision making (QDM) are required. Linguistic information, taking the form of natural or artificial languages, is a natural way to represent qualitative information [2]. In the fuzzy linguistic approaches, linguistic expressions are modeled and computed based on specific linguistic models. The traditional linguistic models include the extension principle-based model [3], the ordered structured model [4], [5], the linguistic 2-tuple model [6] and the virtual linguistic model [7], [8]. By defining syntax and semantics

This work was supported in part by the National Natural Science Foundation of China (Nos. 71571123, 71601092, 61640220), the Key University Science Research Project of Jiangsu Province (No. 16KJA520002), and the Government Audit Research Foundation of Nanjing Audit University

H. Wang is with the School of Information Engineering, Nanjing Audit University, Nanjing, Jiangsu 211815, China (e-mail: wanghai@nau.edu.cn).

Z. S. Xu is with the Business School, Sichuan University, Chengdu, Sichuan 610064, China (e-mail: xuzeshui@263.com).

X-J. Zeng is with the School of Computer Science, University of Manchester, Manchester M13 9PL, UK (e-mail: x.zeng@manchester.ac.uk).

H. C. Liao is with the Business School, Sichuan University, Chengdu, Sichuan 610064, China (e-mail: liaohuchang@163.com). of each linguistic term in a predefined domain, these models represent linguistic information by single terms. For example, the noise of an engine could be "low".

However, the individual granularity of knowledge may not coincide with the granularity of the predefined linguistic term set (LTS). There are some techniques for this case. Unbalanced LTSs [9] are usually considered if objects are not uniformly distributed in the reference domain. Multi-granular LTSs [10] are developed to present different granularities to meet individual granularities of knowledge. Besides, the use of single terms might not be sufficiently convenient to express opinions. In this case, experts may hesitate and balance among several linguistic terms and then seek for complex linguistic expressions (CLEs) which match their opinions precisely. Roughly, CLEs refer to the linguistic expressions, including or implying more than one linguistic term, organized by natural or artificial languages. Till now, there are four techniques focusing on the CLEs which are expressed by natural languages, i.e., uncertain linguistic terms (ULTs) [11], hesitant fuzzy linguistic term sets (HFLTSs) [12], [13], linguistic expressions based on label semantics [14], and linguistic terms with weakened hedges (LTWHs) [15]. ULTs are suitable for the case when the value of a linguistic variable locates between two linguistic terms. As a popular topic, HFLTSs are a powerful tool to model comparative linguistic expressions. Different from the traditional fuzzy linguistic approach, the label semantics model is another methodology to represent several kinds of natural linguistic expressions in term of label descriptions, appropriateness measures and mass assignments. Recently, we highlighted the use of weakened hedges, such as "more or less", to express the uncertainty of using single linguistic terms and presented a new linguistic model of LTWHs [15]. A LTWH incorporates multiple linguistic terms by using weakened hedges. For instance, when evaluating the noise of an engine, one may be not sure whether "low" is the most appropriate term. He/she may state that it is "more or less low". Here, the hedge does not modify the term to another. It means that other terms which are close to the term "low" are also possible. From the perspective of fuzzy set theory, "low" is a fuzzy subset of "more or less low".

LTWHs present a natural manner to express the uncertainty of using single terms. In this paper, we devote to developing the application of LTWHs under the framework of QDM with preference relations. It is widely acknowledged that preference relations play an important role in relative measurement problems [16], [17]. In this setting, decision information is collected by pairwise comparisons between any two objects. However, uncertainties are generally unavoidable when ex- 
pressing the comparisons between two objects by selecting proper linguistic terms from a LTS. Experts would like to seek for CLEs in natural languages, such as ULTs, HFLTSs, and LTWHs, to represent the uncertainty. Till now, several categories of preference relations have been proposed and developed for QDM problems, such as linguistic preference relations (LPRs) [18], [19], uncertain LPRs (ULPRs) [20], and hesitant fuzzy LPRs (HFLPRs) [21], [22]. It is clear that the CLEs taking the form of LTWHs are as natural as comparative linguistic expressions. Using weakened hedges corresponds with the natural language convention to express uncertain preferences. Therefore, in this paper, we present a new type of preference relations, namely linguistic preference relations with weakened hedges (LHPRs), whose entries take the form of LTWHs.

Consistency measures are always considered to guarantee that a preference relation is logically correct [23]. Associated with specific types of preference relations, consistency measures can be defined by means of transitivity, such as additive transitivity, multiplicative transitivity and weak transitivity [24]-[26]. Besides, the degree of consistency is also frequently employed to ensure that a preference relation is with satisfactory consistency [19], [27]. Motivated by the existing strategies of defining consistency measures, this paper systematically investigates several categories of consistency measures of LHPRs. Based on a set of predefined basic operations, equivalent relations and partial orders, some consistency measures are proposed by defining transitivity in the circumstance of LTWHs. The properties of these measures, relationship among these measures and judgement theorems are also investigated. Especially, the theory and algorithms of checking weak consistency and additive consistency are developed. If a LHPR is not weakly consistent, or if the degree of additive consistency is not satisfactory, specific algorithms are proposed to improve its consistency.

The innovation of this paper can be summarized as follows:

(1) The theoretical foundation of applying LHPRs in QDM is developed. The definition of LHPRs is presented based on a new computational model. Especially, the identification and improvement of weak consistency and additive consistency are deeply studied. Therefore, the exploitation of priorities from LHPRs can be further designed based on the theoretical framework presented in this paper.

(2) Definitions and theories related to LHPRs are developed and demonstrated in a visible manner by using the graph theory. Different from the common way of using graphs in preference relations, such as in [22], [23], [28], we utilize the fuzzy weighted digraphs whose weights are fuzzy sets rather than numerical values. Specifically, the weights of arcs in a preference graph are defined by LTWHs. The use of fuzzy or linguistic information enables a flexible way to model the uncertainty in pair-wise comparisons.

(3) When considering the consistency improving, the deviation between two LHPRs, the degree of additive consistency and the threshold of satisfactory consistency are represented by linguistic information. Compared with the traditional strategy which quantifies the degree by numbers in $[0,1]$, the linguistic threshold can be determined by decision-makers in an easy and interpretable way.

Following the above introduction, the rest part of the paper is organized as follows: Section II recalls the representational model of LTWHs and defines some basic operations, equivalent realtions and partial orders. The concepts of LHPRs, as well as preference graphs, are presented in Section III. The concepts and properties of consistencies of LHPRs are then defined and developed in Section IV. Thereafter Section V is devoted to improving LHPRs to satisfy certain consistency measures. A real case study and comparisons are presented in Section VI. The paper is ended by Section VII with some conclusions.

\section{LINGUISTIC TERMS WITH WEAKENED HEDGES}

We will recall the syntax and semantics of LTWHs, and then propose some basic operations associated with properties and partial orders in this section.

\section{A. Syntax and semantics of LTWHs}

The key idea of computing with words is the use of linguistic variables to manage and model the inherent vagueness and uncertainty of the linguistic descriptors. In traditional fuzzy linguistic approaches, the values of a linguistic variable are collected by a LTS in which each linguistic term is associated with its syntax and semantics. For the convenience of expressing preferences of pairwise comparisons, the following commonly use subscript-symmetric version of LTS, defined in the reference domain $U=[L, R]$, is considered [7]:

$$
S=\left\{s_{\alpha} \mid \alpha=-\tau, \ldots, 0, \ldots, \tau\right\}
$$

where $s_{0}$ means indifferent preference, $\tau$ is a positive integer and $S$ satisfies:

(1) Total order: $s_{\alpha} \leq s_{\beta} \Leftrightarrow \alpha \leq \beta$;

(2) Negation operator: neg $\left(s_{\alpha}\right)=s_{-\alpha}$.

For convenience, we employ a function $N: S \rightarrow[0,1]$ such that

$$
N\left(s_{\alpha}\right)=(\tau+\alpha) / 2 \tau
$$

to transform $s_{\alpha}$ into a numerical value. Its inverse function is denoted as $N^{-1}(x)=s_{\alpha}$, where $\alpha=\tau(2 x-1)$.

If an expert is not sure about a linguistic term, then weakened hedges can be considered. Generally, for a specific QDM problem in hand, a set of weakened hedges can be collected based on the language convention of the involved experts and linguistic knowledge at first, and then encoded and classified according to their weakening power. Then the hedges are generally selected according to the degree of uncertainty. Formally, a weakened hedge set (WHS) can be defined for a QDM problem as follows [15]:

$$
H=\left\{h_{t} \mid t=1,2, \ldots, \zeta\right\}
$$

where $h_{j}$ has more weakening force than $h_{i}$ if and only if $i<j$.

Remark 1: In fact, the correspondence between hedges in natural languages and $h_{t} \in H$ may be not one-to-one. This is because we may use different hedges to represent the same or indistinguishable weakening power. For example, one may 
think that the weakening power of "rather" and "roughly" are nearly the same. In this case, the set of hedges with the same or indistinguishable weakening power are encoded by the same $h_{t}$. In addition, the value of $\zeta$ should be relatively small, such as $\zeta \leq 3$, in applications. Because using too many hedges may lead to the difficulty of distinguishing their semantics.

Due to our language convention, we may use some hedges which have no weakening power. For example, we may say "really low" or "definitely low". Here, the hedges "really" and "definitely" imply that one is sure that "low" is an appropriate term to measure the object. In this sense, single terms can be regarded as the special cases of LTWHs. Thus Eq. (3) can be extended as [15]:

$$
\bar{H}=\left\{h_{t} \mid t=0,1,2, \ldots, \zeta\right\}
$$

where $h_{0}$ denotes the hedge(s) with no weakening power.

LTWHs can be generated based on a LTS $S$ and a WHS $H$ as follows [15]:

Definition 1: Given a LTS $S$ and a WHS $H$ defined as before, a LTWH, denoted by $l$, is generated by the following syntactic rule:

$$
\begin{gathered}
\langle\text { weakened hedge }\rangle:=h_{t}, h_{t} \in H, \\
\langle\text { atomic term }\rangle:=s_{\alpha}, s_{\alpha} \in S, \\
\langle L T W H:=\langle\text { weakened hedge }\rangle\langle\text { atomic term }\rangle,
\end{gathered}
$$

which can be represented by a 2-tuple, i.e., $l=\left\langle h_{t}, s_{\alpha}\right\rangle$.

Formally, an atomic term $s_{\alpha} \in S$ can be seen as a special case of LTWH. That is, $s_{\alpha}=\left\langle h_{0}, s_{\alpha}\right\rangle$. This means that LTWHs are a generalization of single linguistic terms.

The classification and encoding of hedges could limit the number of hedges to be represented and thus reduce the complexity of the model. Moreover, we assume that the gaps of weakening power between $h_{t}$ and $h_{t+1}$ are equal, for any $t=0,1, \ldots, \zeta-1$. Therefore, it is natural to define the semantics of $\left\langle h_{1}, s_{\alpha}\right\rangle$ based on that of $\left\langle h_{0}, s_{\alpha}\right\rangle$. This is defined as follows [15]:

Definition 2: Let $S$ be a LTS defined in $U$. For any $x \in U$, $\mu_{\left\langle h_{1}, s_{\alpha}\right\rangle}(x)$ is defined by

$$
\mu_{\left\langle h_{1}, s_{\alpha}\right\rangle}(x)=\sup _{y \in U} \mathcal{T}\left(\operatorname{Sim}(x, y), \mu_{s_{\alpha}}(y)\right)
$$

where $\operatorname{Sim}$ is the similarity measure of $x, y \in U, \mu_{s_{\alpha}}$ is the membership function of $s_{\alpha}$, and $\mathcal{T}$ is a triangular norm.

In order to specify the semantics of $\left\langle h_{1}, s_{\alpha}\right\rangle$, we specify the semantics of atomic terms in a usual way. Triangular fuzzy numbers (TFNs) defined in the given domain $U$ are usually considered to depict the semantics of linguistic terms. Given a TFN $(a, b, c)$, we have:

$$
\mu_{(a, b, c)}(x)=\left\{\begin{array}{cc}
(x-a) /(b-a), & \max \{L, a\} \leq x<b \\
(c-x) /(c-b), & b \leq x \leq \min \{c, R\} \\
0, & \text { otherwise }
\end{array}\right.
$$

Specifically, given a reference domain $U$, we assume that the semantics is defined by two steps: (1) insert $2 \tau+1$ points $\left\{x_{i} \mid i=-\tau, \ldots, 0, \ldots, \tau\right\}$ into the domain $U$ such that $x_{-\tau}=$ $L, x_{\tau}=R$, and $x_{i}<x_{j}$ for any $i<j$; (2) consider two sets

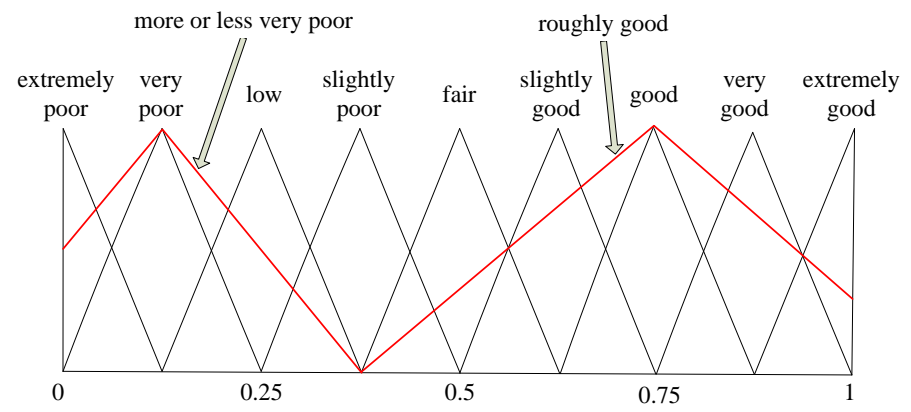

Fig. 1. An example of LTS defined in uniformly distributed domain and examples of semantics of LTWHs

of points, which are out of the domain, denoted as $\left\{x_{-\tau-i} \mid i=\right.$ $1,2, \ldots, \zeta+1\}$ and $\left\{x_{\tau+j} \mid j=1,2, \ldots, \zeta+1\right\}$, where $\zeta$ is the number of hedges considered in Eq. (3). Then the semantics of $s_{\alpha}$ can be represented by a TFN $\left(x_{\alpha-1}, x_{\alpha}, x_{\alpha+}\right)$. Moreover, the domain $U$ is called to be piecewise uniformly distributed because the membership function of each atomic term is a piecewise linear function.

Frequently, like the cases in Fig. $1,\left|x_{i}-x_{i-1}\right|=\mid x_{j}-$ $x_{j-1} \mid=\delta$ holds for any $i, j=-\tau-\zeta, \ldots, 0, \ldots, \tau+\zeta+1$. Then $U$ is said to be uniformly distributed. In this case, the similarity of $x$ and $y$ can be defined based on their distance [15]. Given $x, y \in U$,

$$
\operatorname{Sim}(x, y)=\left\{\begin{array}{cc}
1-|x-y| / \delta, & |x-y|<\delta \\
0, & \text { otherwise }
\end{array}\right.
$$

Moreover, if $\mathcal{T}(x, y)=\min \{x, y\}$, it can be concluded that $\mu_{\left\langle h_{1}, s_{\alpha}\right\rangle}=\left(x_{\alpha-2}, x_{\alpha}, x_{\alpha+2}\right)$. As it has been assumed that the gaps of weakening power of any two adjacent hedges are equal, the conclusion can be generalized as $\mu_{\left\langle h_{t}, s_{\alpha}\right\rangle}=$ $\left(x_{\alpha-t-1}, x_{\alpha}, x_{\alpha+t+1}\right)$, where $h_{t} \in \bar{H}, s_{\alpha} \in S$ [15].

Generally, if $U$ is piecewise uniformly distributed, a piecewise linear function can be employed to transform $U$ into an uniformly distributed domain $[0,1]$. The above conclusion holds as well. Thus, we have the following theorem [15]:

Theorem 1: Let $S$ and $\bar{H}$ be defined as before, $\mathcal{T}(x, y)=$ $\min \{x, y\}$. For any $s_{\alpha} \in S$ and $h_{t} \in \bar{H}$, the semantics of $\left\langle h_{t}, s_{\alpha}\right\rangle$ is

$$
\mu_{\left\langle h_{t}, s_{\alpha}\right\rangle}=\left(x_{\alpha-t-1}, x_{\alpha}, x_{\alpha+t+1}\right)
$$

Example 1: Given a LTS $S=\left\{s_{-4}=\right.$ extremely poor, $s_{-3}=$ very poor, $s_{-2}=$ poor, $s_{-1}=$ slightly poor, $s_{0}=$ indifferent, $s_{1}=$ slightly good, $s_{2}=$ good, $s_{3}=$ very good, $s_{4}=$ extremely good $\}$ and a WHS $\bar{H}=\left\{h_{0}=\right.$ definitely, $h_{1}=$ more or less, $h_{2}=$ roughly $\}$, three LTWHs might be:

$$
\begin{gathered}
\left\langle h_{0}, s_{0}\right\rangle: \text { (definitely) fair; } \\
\left\langle h_{1}, s_{-3}\right\rangle: \text { more or less very poor } \\
\left\langle h_{2}, s_{2}\right\rangle: \text { roughly good }
\end{gathered}
$$

the semantics of $\left\langle h_{1}, s_{-3}\right\rangle$ and $\left\langle h_{2}, s_{2}\right\rangle$ can be found in Fig. 1 .

Remark 2: As can be seen in Example 1, there are two hedges, i.e., "more or less" and "very", in $\left\langle h_{1}, s_{-3}\right\rangle$. But there 
are some differences. Firstly, "more or less" is a weakened hedge whereas "very" is an intensified hedge. As we focus on the model of uncertainty of using single terms, the intensified hedges are not necessary. Secondly, "very" in this case acts as a non-inclusive hedge whereas "more or less" is an inclusive hedge. See [29] for more details about these two explanations. Here, "very" moves "poor" to a completely different term. However, "very poor" is a fuzzy subset of "more or less very poor".

The characteristics of the representational model are distinctive, compared with other techniques for CLEs in natrual languages. The approach to represent the uncertainty of selecting linguistic terms is unique. In fact, CLEs are considered generally because we cannot determine which linguistic term is the most appropriate to describe the objects. Thus, if the boundary terms emerge in our mind, then it is natural to use them and the connectives, such as "between", "and", to compose ULTs or HFLTSs to describe the linguistic opinion. If a single linguistic term might be the real value of a linguistic variable, but we are not confident enough to confirm the assertion, then according to the language convention, the natural way is to consider a weakened hedge to express the degree of uncertainty so that the expressed linguistic information is more confident. Accordingly, from the perspective of syntactic rules, the elements of generating LTWHs are different from others. Specifically, a LTWH is formed by a weakened hedge and an atomic term. Connectives are thus not necessary. As has been pointed out in [30], modelling the power of hedges is very complicated. Our specified semantics of hedges is just a possible and rational solution. We consider this strategy because: (1) the semantics can be determined only by the distribution of the domain, which has been fixed as long as the LTS is provided; and (2) the result shown in Theorem 1 is compact and concise.

Given $S$ defined in Eq.(1) and $\bar{H}$ in Eq.(4), the set of all LTWHs is denoted by $\mathcal{L}$ in this paper. Moreover, in the virtual linguistic model, $S$ is extended to a continuous version $\bar{S}=$ $\left\{s_{\alpha} \mid \alpha \in[-q, q], q>\tau\right\}$. For the convenience of computation, a 2-tuple $\left\langle h_{t}, s_{\alpha}\right\rangle$, where $s_{\alpha} \in \bar{S}$, is also called a LTWH. The set of all LTWHs, associate with $\bar{S}$ and $\bar{H}$ defined as above, is denoted by $\overline{\mathcal{L}}$ in the sequel. Given $\left\langle h_{t}, s_{\alpha}\right\rangle \in \overline{\mathcal{L}}$, if $\left\langle h_{t}, s_{\alpha}\right\rangle \notin \mathcal{L}$, then it is called a virtual LTWH which only appears in the process of computation.

\section{B. Basic operations and order relations of LTWHs}

The negation operator can be defined naturally based on the negation operator recalled above [15].

Definition 3: Given $l=\left\langle h_{t}, s_{\alpha}\right\rangle \in \overline{\mathcal{L}}$, its negation is defined by:

$$
N e g\left(\left\langle h_{t}, s_{\alpha}\right\rangle\right)=\left\langle h_{t}, \operatorname{neg}\left(s_{\alpha}\right)\right\rangle=\left\langle h_{t}, s_{-\alpha}\right\rangle
$$

According to the semantics of LTWHs, the term $s_{\alpha}$ in $\left\langle h_{t}, s_{\alpha}\right\rangle$ somewhat acts as the expectation of $\left\langle h_{t}, s_{\alpha}\right\rangle$. This motivates us to define the following strictly partial order $\prec$ :

Definition 4: Given two LTWHs $l_{1}=\left\langle h_{t_{1}}, s_{\alpha_{1}}\right\rangle, l_{2}=$ $\left\langle h_{t_{2}}, s_{\alpha_{2}}\right\rangle \in \overline{\mathcal{L}}$, then

$$
l_{1} \prec l_{2} \Leftrightarrow s_{\alpha_{1}}<s_{\alpha_{2}}
$$

If $s_{\alpha_{1}}=s_{\alpha_{2}}$, then $l_{1}$ and $l_{2}$ are incomparable according to Definition 4. In fact, this can be depicted by the following equivalent relation.

Definition 5: Given two LTWHs $l_{1}=\left\langle h_{t_{1}}, s_{\alpha_{1}}\right\rangle, l_{2}=$ $\left\langle h_{t_{2}}, s_{\alpha_{2}}\right\rangle \in \overline{\mathcal{L}}$, then

$$
l_{1} \simeq l_{2} \Leftrightarrow s_{\alpha_{1}}=s_{\alpha_{2}}
$$

Moreover, given two matrices $P_{1}$ and $P_{2}$, the notation $P_{1} \simeq$ $P_{2}$ means that their corresponding entries are equivalent in the sense of Definition 5. Notice that two binary relations, i.e., = and $\simeq$, will be used in the sequel. $\left\langle h_{t_{1}}, s_{\alpha_{1}}\right\rangle=\left\langle h_{t_{2}}, s_{\alpha_{2}}\right\rangle$ always means $t_{1}=t_{2}$ and $\alpha_{1}=\alpha_{2}$. Therefore, $l_{1}=l_{2}$ always implies $l_{1} \simeq l_{2}$. Eqs. (10-11) implies a poset $(\overline{\mathcal{L}}, \preceq)$, where $\preceq$ is defined by

$$
l_{1} \preceq l_{2} \Leftrightarrow\left(l_{1} \prec l_{2}\right) \vee\left(l_{1} \simeq l_{2}\right)
$$

for any $l_{1}, l_{2} \in \overline{\mathcal{L}}$. For convenience, if $l_{1} \preceq l_{2}$ (or $l_{1} \prec l_{2}$ ), we also write $l_{2} \succeq l_{1}$ (or $l_{2} \succ l_{1}$ ).

To ease the development of LHPRs, the following basic operations are necessary.

Definition 6: Given three LTWHs $l=\left\langle h_{t}, s_{\alpha}\right\rangle, l_{1}=$ $\left\langle h_{t_{1}}, s_{\alpha_{1}}\right\rangle, l_{2}=\left\langle h_{t_{2}}, s_{\alpha_{2}}\right\rangle \in \overline{\mathcal{L}}, \lambda>0$, we define

(1) $l_{1} \oplus l_{2}=\left\langle h_{\max \left\{t_{1}, t_{2}\right\}}, s_{\alpha_{1}+\alpha_{2}}\right\rangle$;

(2) $\lambda l=\left\langle h_{t}, s_{\lambda \alpha}\right\rangle$;

(3) $l_{1} \otimes l_{2}=\left\langle h_{\max \left\{t_{1}, t_{2}\right\}}, N^{-1}\left(N\left(s_{\alpha_{1}}\right) \cdot N\left(s_{\alpha_{2}}\right)\right)\right\rangle$.

Apparently, if $h_{t}=h_{t_{1}}=h_{t_{2}}=h_{0}$ in Definition 6, then the operations are reduced to the version defined in [7]. For the convenience of representation in some cases, we introduce the subtraction of two LTWHs. Given $l_{1}, l_{2}, l_{3} \in \overline{\mathcal{L}}$, if $l_{2} \oplus l_{3}=l_{1}$, then we denote $l_{3}=l_{1} \ominus l_{2}$. Associated with the operation $\oplus$, we obtain:

$$
l_{1} \ominus l_{2}=\left\langle h_{\max \left\{t_{1}, t_{2}\right\}}, s_{\alpha_{1}-\alpha_{2}}\right\rangle
$$

The following properties are required in the sequel.

Theorem 2: For any $l, l_{1}, l_{2}, l_{3} \in \overline{\mathcal{L}}, \lambda_{1}, \lambda_{2}>0$, we have

(1) $N e g(N e g(l))=l, N e g(l)=s_{0} \ominus l$;

(2) $l \oplus s_{0}=l, l \ominus s_{0}=l$;

(3) Associative: $l_{1} \oplus l_{2} \oplus l_{3}=l_{1} \oplus\left(l_{2} \oplus l_{3}\right), l_{1} \ominus l_{2} \ominus l_{3}=$ $l_{1} \ominus\left(l_{2} \oplus l_{3}\right)$

(4) Distributive: $\lambda\left(l_{1} \oplus l_{2}\right)=\left(\lambda l_{1}\right) \oplus\left(\lambda l_{2}\right),\left(\lambda_{1}+\lambda_{2}\right) l=$ $\left(\lambda_{1} l\right) \oplus\left(\lambda_{2} l\right)$;

(5) $N e g\left(l_{1}\right) \oplus N e g\left(l_{2}\right)=N e g\left(l_{1} \oplus l_{2}\right)$; where $s_{0}=\left\langle h_{0}, s_{0}\right\rangle$ is the middle element in $\bar{S}$.

The proof of this properties is straightforward according to the above definitions. Moreover, different from the idea of many references, such as [19], which uses a number in $[0,1]$ to represent the deviation between two linguistic terms in $\bar{S}$, we measure the deviation between two LTWHs in $\overline{\mathcal{L}}$ by means of a LTWH as follows:

Definition 7: Given $l_{1}=\left\langle h_{t_{1}}, s_{\alpha_{1}}\right\rangle, l_{2}=\left\langle h_{t_{2}}, s_{\alpha_{2}}\right\rangle \in \overline{\mathcal{L}}$, the deviation between $l_{1}$ and $l_{2}$ is defined by:

$$
d\left(l_{1}, l_{2}\right)=\left\{\begin{array}{l}
l_{1} \ominus l_{2}, \quad l_{1} \succeq l_{2} \\
l_{2} \ominus l_{1}, \quad \text { otherwise }
\end{array}\right.
$$

The linguistic form of deviations make use of LTWHs in $\left\{\left\langle h_{t}, s_{\alpha}\right\rangle \mid h_{t} \in \bar{H}, s_{\alpha} \in \bar{S}\right.$ and $\left.s_{\alpha} \succeq s_{0}\right\}$ to represent the gap 
between two LTWHs. The uncertainty implied by the hedges of the two LTWHs is remained in the deviation measure. For instance, let $l_{1}=s_{1}$ and $l_{2}=\left\langle h_{1}, s_{1}\right\rangle$ be two LTWHs based on $S$ and $\bar{H}$ specified in Example 1 , then $d\left(l_{1}, l_{2}\right)=\left\langle h_{1}, s_{0}\right\rangle$, which means that the deviation between "slightly good" and "more or less slightly good" is "more or less indifferent". The deviation measure possesses some interesting properties.

Theorem 3: Let $l_{1}=\left\langle h_{t_{1}}, s_{\alpha_{1}}\right\rangle, l_{2}=\left\langle h_{t_{2}}, s_{\alpha_{2}}\right\rangle \in \overline{\mathcal{L}}$, we have

(1) $d\left(l_{1}, l_{2}\right)=\left\langle h_{\max \left\{t_{1}, t_{2}\right\}}, s_{\left|\alpha_{1}-\alpha_{2}\right|}\right\rangle$;

(2) If $l_{1} \simeq l_{2}$, then $d\left(l_{1}, l_{2}\right) \simeq s_{0}$;

(3) If $l_{1} \simeq l_{2}$, then $d\left(l, l_{1}\right) \simeq d\left(l, l_{2}\right)$ for any $l \in \overline{\mathcal{L}}$;

(4) $d\left(l_{1}, l\right) \oplus d\left(l, l_{1}\right) \succeq d\left(l_{1}, l_{2}\right)$ for any $l \in \overline{\mathcal{L}}$.

Proof. Let $l=\left\langle h_{t}, s_{\alpha}\right\rangle \in \overline{\mathcal{L}}$,

(1) If $l_{1} \succeq l_{2}, d\left(l_{1}, l_{2}\right)=l_{1} \ominus l_{2}=\left\langle h_{\max \left\{t_{1}, t_{2}\right\}}, s_{\alpha_{1}-\alpha_{2}}\right\rangle$; if $l_{2} \succeq l_{1}$, then $d\left(l_{1}, l_{2}\right)=l_{2} \ominus l_{1}=\left\langle h_{\max \left\{t_{1}, t_{2}\right\}}, s_{\alpha_{2}-\alpha_{1}}\right\rangle$. Thus $d\left(l_{1}, l_{2}\right)=\left\langle h_{\max \left\{t_{1}, t_{2}\right\}}, s_{\left|\alpha_{1}-\alpha_{2}\right|}\right\rangle$.

(2)-(3). If $l_{1} \simeq l_{2}$, then we have $s_{\alpha_{1}}=s_{\alpha_{2}} . d\left(l_{1}, l_{2}\right)=$ $\left\langle h_{\max \left\{t_{1}, t_{2}\right\}}, s_{0}\right\rangle \simeq s_{0} ; d\left(l, l_{1}\right)=\left\langle h_{\max \left\{t, t_{1}\right\}}, s_{\left|\alpha-\alpha_{1}\right|}\right\rangle=$ $\left\langle h_{\max \left\{t, t_{1}\right\}}, s_{\left|\alpha-\alpha_{2}\right|}\right\rangle \simeq\left\langle h_{\max \left\{t, t_{2}\right\}}, s_{\left|\alpha-\alpha_{2}\right|}\right\rangle=d\left(l, l_{2}\right)$.

(4) $d\left(l_{1}, l\right) \oplus d\left(l, l_{2}\right)=\left\langle h_{\max \left\{t, t_{1}\right\}}, s_{\left|\alpha-\alpha_{1}\right|}\right\rangle \oplus$ $\left\langle h_{\max \left\{t, t_{2}\right\}}, s_{\left|\alpha-\alpha_{2}\right|}\right\rangle=\left\langle h_{\max \left\{t, t_{1}, t_{2}\right\}}, s_{\left|\alpha-\alpha_{1}\right|+\left|\alpha-\alpha_{2}\right|}\right\rangle \succeq$ $\left\langle h_{\max \left\{t, t_{1}, t_{2}\right\}}, s_{\left|\alpha_{1}-\alpha_{2}\right|}\right\rangle \simeq d\left(l_{1}, l_{2}\right)$.

\section{LINGUISTIC PREFERENCE RELATIONS WITH WEAKENED HEDGES}

This section is devoted to defining the concept of LHPRs and presenting a graphical view for further processing. When expressing preference degrees over a set of objects, weakened hedges would be quite natural to be considered if the expert is not confident enough to use a certain term. This leads to the appearance of LTWHs in preference relations. Thus, we present the following definition:

Definition 8: Let $\bar{S}$ and $\bar{H}$ be defined as before. A binary relation $P$ defined on the set $V=\left\{v_{1}, v_{2}, \ldots, v_{n}\right\}$ is called a LHPR if, for any $i, j=1,2, \ldots, n, P\left(v_{i}, v_{j}\right)=l_{i j} \in \overline{\mathcal{L}}$ represents the degree of $v_{i}$ being preferred to $v_{j}$. Formally, a LHPR is denoted by $P=\left(l_{i j}\right)_{n \times n}=\left(\left\langle h_{t_{i j}}, s_{\alpha_{i j}}\right\rangle\right)_{n \times n}$, where $h_{t_{i j}} \in \bar{H}, s_{\alpha_{i j}} \in \bar{S}$. $P$ is said to be reciprocal if $l_{i j}=N e g\left(l_{j i}\right)$ for any $i, j=1,2, \ldots, n$.

Two conclusions are transparent: (1) If $t_{i j}=0$, i.e., $h_{t_{i j}}=$ $h_{0}$, for any $i, j=1,2, \ldots, n$, then the LHPR is reduced to a traditional LPR; (2) For any $i=1,2, \ldots, n, l_{i i} \simeq\left\langle h_{0}, s_{0}\right\rangle$. In fact, $l_{i i}=N e g\left(l_{i i}\right)$ means $\left\langle h_{t_{i i}}, s_{\alpha_{i i}}\right\rangle=\left\langle h_{t_{i i}}, s_{-\alpha_{i i}}\right\rangle$. Thus $\alpha_{i i}=-\alpha_{i i}$, which results in $\alpha_{i i}=0$. Notice that we use $l_{i i} \simeq s_{0}$ instead of the traditional form $l_{i i}=s_{0}$. This is just a theoretical generalization for the convenience of inducing some necessary properties. The generalization does not mean that there exists any uncertainty in self comparisons.

In this paper, the graph theory is considered to handle consistency measures of LHPRs in a visible manner. Generally, a digraph $G$ can be defined by a 2-tuple $G=(V, A)$ where $V$ is the set of vertices and $A$ is the set of arcs. In a fuzzy weighted graph [31], the vertices and arcs (or edges) are crisp, but the weights of arcs (or edges) are fuzzy. In this study, we specify the weights of arcs by LTWHs which come from the entries of a LHPR. The LHPR is visualized by its associated fuzzy weighted digraph.

Definition 9: Let $P=\left(l_{i j}\right)_{n \times n}$ be a LHPR defined on $V=\left\{v_{1}, v_{2}, \ldots, v_{n}\right\}$. A preference relation graph (P-graph) associated with $P$ is a fuzzy weighted digraph $G_{P}=(V, A)$, where the set of vertices is $V$, the set of arcs is denoted by $A=\left\{\left(v_{i}, v_{j}\right)\right\}$, and the $\operatorname{arc}\left(v_{i}, v_{j}\right)$ which joins $v_{i}$ to $v_{j}$ exists if $l_{i j} \succeq s_{0}$ and $i \neq j$. If $\left(v_{i}, v_{j}\right)$ exists, then its fuzzy weight is defined by $w\left(v_{i}, v_{j}\right)=l_{i j}$.

In a P-graph, the arc $\left(v_{i}, v_{j}\right)$ indicates that $v_{i}$ is preferred to $v_{j}$. Preference values which are equivalent to $s_{0}$ are considered as a special case of preferences. Given a LHPR $P\left(l_{i j}\right)_{n \times n}$, the number of arcs is $n(n-1) / 2+c_{0}$, where $c_{0}$ is the number of upper diagonal entries which are equivalent to $s_{0}$. The following digraph is specified to highlight each entry of $P$ :

Definition 10: Let $P=\left(l_{i j}\right)_{n \times n}$ be a LHPR defined on $V=\left\{v_{1}, v_{2}, \ldots, v_{n}\right\}$. A symmetric preference relation graph (SP-graph) associated with $P$ is fuzzy weighted digraph $G_{P}=$ $(V, A)$, where the set of vertices is $V$, the set of edges is denoted by $A=\left\{\left(v_{i}, v_{j}\right)\right\}$, and the $\operatorname{arc}\left(v_{i}, v_{j}\right)$ joins $v_{i}$ to $v_{j}$ with its fuzzy weight $w\left(v_{i}, v_{j}\right)=l_{i j}, i \neq j$.

Different from P-graphs, there are $n(n-1)$ arcs in a SP-graph associated with $P=\left(l_{i j}\right)_{n \times n}$. In the Pgraph (or SP-graph) $G_{P}$ associated with the LHPR $P$, a sequence $\left(v_{i_{1}},\left(v_{i_{1}}, v_{i_{2}}\right), v_{i_{2}}, \ldots, v_{i_{k-1}},\left(v_{i_{k-1}}, v_{i_{k}}\right), v_{i_{k}}\right)$ is a walk from $v_{i_{1}}$ to $v_{i_{k}}$, where $v_{i_{1}}, v_{i_{2}}, \ldots, v_{i_{k}} \in V$ and $\left(v_{i_{1}}, v_{i_{2}}\right), \ldots,\left(v_{i_{k-1}}, v_{i_{k}}\right) \in A$. A walk is called a path if $v_{i_{1}}, v_{i_{2}}, \ldots, v_{i_{k}}$ are distinct. The fuzzy length of the path is defined by the sum of weights of its arcs, i.e., $\operatorname{len}\left(v_{i_{1}},\left(v_{i_{1}}, v_{i_{2}}\right), v_{i_{2}}, \ldots, v_{i_{k-1}},\left(v_{i_{k-1}}, v_{i_{k}}\right), v_{i_{k}}\right)=l_{i_{1} i_{2}} \oplus$ $\cdots \oplus l_{i_{k-1} i_{k}}$ The fuzzy distance from $v_{i}$ to $v_{j}$ is the shortest fuzzy length of the paths from $v_{i}$ to $v_{j}$ in $G_{P}$. If $v_{i_{1}}=v_{i_{k}}$ and $v_{i_{1}}, v_{i_{2}}, \ldots, v_{i_{k-1}}$ are distinct, then a walk is a cycle.

Traditionally, the arcs in a digraph $G$ with $n$ vertices are represented by an adjacent matrix $M=\left(M_{i j}\right)_{n \times n}$, having $M_{i j}=1$ if and only if there is an $\operatorname{arc}\left(v_{i}, v_{j}\right)$. Given a LHPR $P=\left(l_{i j}\right)_{n \times n}$, the arcs of its associated P-graph $G_{P}$ can be represented by the adjacent metrix $M_{P}=\left(M_{i j}\right)_{n \times n}$, where

$$
M_{i j}= \begin{cases}1, & w\left(v_{i}, v_{j}\right) \succeq s_{0} \\ 0, & w\left(v_{i}, v_{j}\right) \prec s_{0}\end{cases}
$$

According to Definition 9, $M_{i i}=0$ for any $i=1,2, \ldots, n$. Moreover, if $l_{i j} \simeq s_{0}, i \neq j$, then $M_{i j}=M_{j i}=1$. In this sense, the adjacent matrix of the SP-graph of a LHPR is not so interesting because all its off-diagonal elements are equal to 1 .

Example 2: Given $S$ and $\bar{H}$ defined in Example 1, a LHPR $P$ could be:

$\left(\begin{array}{ccccc}s_{0} & \left\langle h_{1}, s_{-2}\right\rangle & \left\langle h_{1}, s_{-3}\right\rangle & s_{-1} & \left\langle h_{2}, s_{2}\right\rangle \\ \left\langle h_{1}, s_{2}\right\rangle & s_{0} & s_{2} & \left\langle h_{1}, s_{1}\right\rangle & s_{2} \\ \left\langle h_{1}, s_{3}\right\rangle & s_{-2} & s_{0} & s_{3} & s_{1} \\ s_{1} & \left\langle h_{1}, s_{-1}\right\rangle & s_{-3} & s_{0} & \left\langle h_{1}, s_{2}\right\rangle \\ \left\langle h_{2}, s_{-2}\right\rangle & s_{-2} & s_{-1} & \left\langle h_{1}, s_{-2}\right\rangle & s_{0}\end{array}\right)$

For example, entry $\left\langle h_{1}, s_{2}\right\rangle$ means that $v_{2}$ is more or less good when compared with $v_{1}$. Its P-graph and SP-graph are shown in Fig.2 and Fig.3, respectively. 


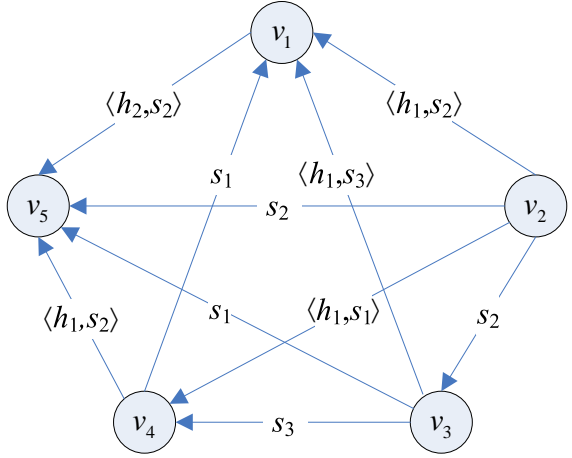

Fig. 2. The P-graph associated with the LHPR in Example 2

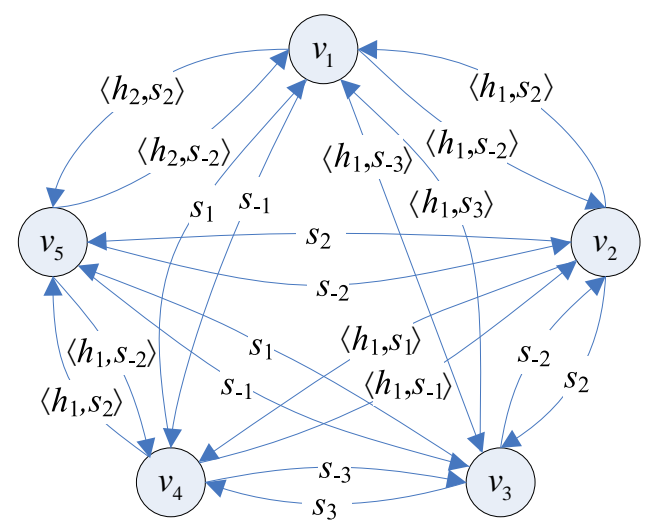

Fig. 3. The SP-graph associated with the LHPR in Example 2

\section{CONSISTENCY MEASURES OF LHPRS}

Based on the concept and the associated P-graph, we now define several kinds of consistency measures to implement the idea of transitivity. The properties, identification and improvement of such measures will be discussed as well.

\section{A. Defining consistencies by transitivity}

Definition 11: Given a reciprocal LHPR $P=\left(l_{i j}\right)_{n \times n}$ with $l_{i j}=\left\langle h_{t_{i j}}, s_{\alpha_{i j}}\right\rangle \in \overline{\mathcal{L}}, i, j=1,2, \ldots, n$, then

(1) $P$ satisfies the triangle condition if $\forall i, j, k=1,2, \ldots, n$,

$$
l_{i k} \oplus l_{k j} \succeq l_{i j}
$$

(2) $P$ satisfies the weak transitivity if $\forall i, j, k=1,2, \ldots, n$,

$$
l_{i k} \succeq s_{0}, l_{k j} \succeq s_{0} \Rightarrow l_{i j} \succeq s_{0}
$$

(3) $P$ satisfies the restricted max-max transitivity if $\forall i, j, k=1,2, \ldots, n$,

$$
l_{i k} \succeq s_{0}, l_{k j} \succeq s_{0} \Rightarrow l_{i j} \succeq \max \left\{l_{i k}, l_{k j}\right\}
$$

(4) $P$ satisfies the restricted max-min transitivity if $\forall i, j, k=1,2, \ldots, n$,

$$
l_{i k} \succeq s_{0}, l_{k j} \succeq s_{0} \Rightarrow l_{i j} \succeq \min \left\{l_{i k}, l_{k j}\right\}
$$

(5) $P$ satisfies the additive transitivity if $\forall i, j, k=$ $1,2, \ldots, n$,

$$
l_{i k} \oplus l_{k j} \simeq l_{i j}
$$

(6) $P$ satisfies the multiplicative transitivity if $\forall i, j, k=$ $1,2, \ldots, n$ and $l_{i j}, l_{j k}, l_{k i} \succ s_{-\tau}$,

$$
l_{i j} \otimes l_{j k} \otimes l_{k i} \simeq l_{i k} \otimes l_{k j} \otimes l_{j i}
$$

Especially, $P$ is said to be weakly consistent, additively consistent and multiplicatively consistent if it satisfies the weak transitivity, additive transitivity and multiplicative transitivity, respectively.

It is very easy to interpret most of the transitivities visually by using P-graphs and SP-graphs. For any $i, j, k=1,2, \ldots, n$, if $v_{i}$ is preferred to $v_{k}$ and $v_{k}$ is preferred to $v_{j}$, then the triangle condition and the weak transitivity stipulate the upper and lower bounds of $w\left(v_{i}, v_{j}\right)$, respectively. Specifically, in a P-graph, if there is an arc directed from $v_{i}$ to $v_{k}$ and another arc directed from $v_{k}$ to $v_{j}$, then the weak transitivity requires that there should be an arc directed from $v_{i}$ to $v_{j}$. Simultaneously, the triangle condition calls for that the fuzzy weight of $\left(v_{i}, v_{j}\right)$ should not exceed the fuzzy length of $\left(v_{i},\left(v_{i}, v_{k}\right), v_{k},\left(v_{k}, v_{j}\right), v_{j}\right)$. As seen in Fig. 2, because there are two arcs $\left(v_{2}, v_{3}\right)$ and $\left(v_{3}, v_{1}\right)$, then there should be an arc $\left(v_{2}, v_{1}\right)$ according to the weak transitivity and $w\left(v_{3}, v_{1}\right)$ should not exceed $w\left(v_{2}, v_{3}\right) \oplus w\left(v_{3}, v_{1}\right)$ according to the triangle condition. From the angle of lower bounds, the restricted max-min transitivity requires that $w\left(v_{i}, v_{j}\right)$ should be no less than $\min \left\{w\left(v_{i}, v_{k}\right), w\left(v_{k}, v_{j}\right)\right\}$; the restricted maxmax transitivity demands that $w\left(v_{i}, v_{j}\right)$ should be at least $\max \left\{w\left(v_{i}, v_{k}\right), w\left(v_{k}, v_{j}\right)\right\}$. The additive transitivity defines $w\left(v_{i}, v_{j}\right)$ by a precise angle, i.e., $w\left(v_{i}, v_{j}\right)$ should be equivalent to $w\left(v_{i}, v_{k}\right) \oplus w\left(v_{k}, v_{j}\right)$ in the sense of Eq.(11). Associated with its SP-graph, a LHPR satisfies the additive transitivity if the fuzzy lengths of all paths which start from $v_{i}$ and end by $v_{j}$ are equivalent. As seen in Fig. 2, $\operatorname{len}\left(v_{2},\left(v_{2}, v_{1}\right), v_{1}\right)=l_{21}=$ $\left\langle h_{1}, s_{2}\right\rangle$, and $\operatorname{len}\left(v_{2},\left(v_{2}, v_{3}\right), v_{3},\left(v_{3}, v_{1}\right), v_{1}\right)=l_{21} \oplus l_{31}=$ $s_{2} \oplus\left\langle h_{1}, s_{3}\right\rangle=\left\langle h_{1}, s_{5}\right\rangle$. Then we have $\left\langle h_{1}, s_{2}\right\rangle \prec\left\langle h_{1}, s_{5}\right\rangle$ and thus $P$ in Example 2 is not additively consistent.

The relationship among the transitivity can be summarized directly based on the definition and the above analyses.

Theorem 4: Given a LHPR $P$ and five propositions: (i) $P$ satisfies the triangle condition; (ii) $P$ satisfies the weak consistency; (iii) $P$ satisfies the restricted max-max transitivity; (iv) $P$ satisfies the restricted max-min transitivity; (v) $P$ satisfies the additive transitivity, then:

(1) $(\mathrm{v}) \Rightarrow$ (i)

(2) (v) $\Rightarrow$ (iii) $\Rightarrow$ (iv) $\Rightarrow$ (ii).

Similar to many studies regarding preference relations, the additive consistency is an ideal property for a LHPR if satisfied. The weak consistency, which ensures that a LHPR is logically correct, is the property that the LHPR should at least satisfy. We mainly focus on these two types of transitivity and start with identifying whether a LHPR is of weak consistency.

\section{B. Weak consistency of LHPRs}

According to the definition, weak transitivity can be checked directly by the P-graph. Similar to Ref. [32], a cycle with $k$ vertices and $k$ arcs is referred to as a $k$-cycle. Weak transitivity requires that there is no $k$-cycle in the P-graph, $3 \leq k \leq n$. Fortunately, it does not have to check all $k \in\{3,4, \ldots, n\}$. 


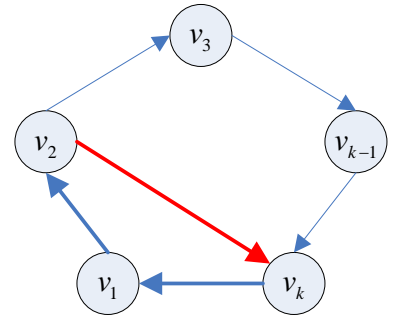

(a) $w\left(v_{2}, v_{k}\right) \succeq s_{0}$

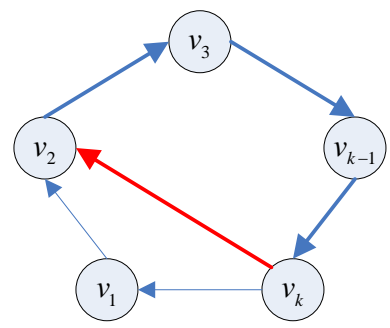

(b) $w\left(v_{k}, v_{2}\right) \succeq s_{0}$

Fig. 4. Illustration of finding 3-cycle from a $k$-cycle. (a) a 3-cycle is found; (b) the $k$-cycle is reduced to a $(k-1)$-cycle.

Theorem 5: For any P-graph associated with a reciprocal LHPR, if there is a $k$-cycle, $k \in\{3,4, \ldots, n\}$, then there is a 3-cycle.

Proof. For any P-garph, according to Definition 9, there is at least one arc located between any two vertices. Given a $k$-cycle denoted by $\left(v_{i_{1}},\left(v_{i_{1}}, v_{i_{2}}\right), v_{i_{2}}, \ldots, v_{i_{k}},\left(v_{i_{k}}, v_{i_{1}}\right), v_{i_{1}}\right)$, where $k \in\{3,4, \ldots, n\}$,

(1) If $w\left(v_{i_{2}}, v_{i_{k}}\right) \succeq s_{0}$, as shown in Fig. 4(a), then there is a 3-cycle $\left(v_{i_{1}},\left(v_{i_{1}}, v_{i_{2}}\right), v_{i_{2}},\left(v_{i_{2}}, v_{i_{k}}\right), v_{i_{k}},\left(v_{i_{k}}, v_{i_{1}}\right), v_{i_{1}}\right)$.

(2) If $w\left(v_{i_{2}}, v_{i_{k}}\right) \prec s_{0}$, according to the reciprocal property, there is an $\operatorname{arc}\left(v_{i_{k}}, v_{i_{2}}\right)$. Ignoring $v_{1}$, we obtain a new $(k-1)$ cycle $\left(v_{i_{2}},\left(v_{i_{2}}, v_{i_{3}}\right), v_{i_{3}}, \ldots, v_{i_{k}},\left(v_{i_{k}}, v_{i_{2}}\right), v_{i_{2}}\right)$, as shown in Fig.4(b). Similarly, when considering $w\left(v_{i_{3}}, v_{i_{k}}\right)$, we could get either a 3 -cycle or a new $(k-2)$-cycle.

Repeating this process, it is apparent that a 3-cycle can be found.

Remark 3: The idea of checking the weak consistency of preference relations has been included in some graph-based approaches, such as [28], [33]. But the fundamental aspect has not been clarified. The proof of Theorem 5 guarantees that this kind of approaches are theoretically correct.

Therefore, it is enough to check all the 3-cycles in a Pgraph for weak consistency judgement. However, not all the 3 -cycles means illogical judgements in a preference relation because the weights of arcs in a 3-cycle might be equivalent to $s_{0}$. The following definition is to identify real cycles.

Definition 12: A $k$-cycle in a P-graph is said to be a strict $k$-cycle if there exists at least one $\operatorname{arc}\left(v_{i}, v_{j}\right)$ whose weight is greater than $s_{0}$, i.e., $w\left(v_{i}, v_{j}\right) \succ s_{0}$.

The definition is straightforward. In fact, if the weight of each arc in a cycle is equivalent to $s_{0}$, then the cycle is meaningless for weak consistency judgement. Formally, we have the following theorem:
Theorem 6: A LHPR is weakly consistent if and only if there is no strict 3-cycle in its associated P-graph.

Proof. The theorem can be rephrased as: A LHPR is not weakly consistent if and only of there exists at least one strict 3 -cycle in its P-graph. Let $P=\left(l_{i j}\right)_{n \times n}$ be a LHPR defined on the set $\left\{v_{1}, v_{2}, \ldots, v_{n}\right\}$, associated with the P-graph $G_{P}$.

$(\Rightarrow)$ If $P$ is not weakly consistent, then there exist $i, j, k \in$ $\{1,2, \ldots, n\}$ such that $l_{i j} \succeq s_{0}, l_{j k} \succeq s_{0}$, and $l_{i k} \prec s_{0}$. Then the 3 -cycle $\left(v_{i},\left(v_{i}, v_{j}\right), v_{j},\left(v_{j}, v_{k}\right), v_{k},\left(v_{k}, v_{i}\right), v_{i}\right)$ always exists.

(1) If $l_{i j} \succ s_{0}$ and $l_{j k} \succ s_{0}$, then the weights of three arcs of the 3-cycle are greater than $s_{0}$. Thus, it is a strict 3-cycle.

(2) If $l_{i j} \succ s_{0}$ and $l_{j k} \simeq s_{0}$, then we have $w\left(v_{i}, v_{j}\right) \succ s_{0}$ and $w\left(v_{k}, v_{i}\right) \succ s_{0}$. It is also a strict 3-cycle.

(3) If $l_{i j} \simeq s_{0}$ and $l_{j k} \simeq s_{0}$, the 3 -cycle is strict because $w\left(v_{k}, v_{i}\right) \succ s_{0}$.

$(\Leftarrow)$ Suppose that there exist $i, j, k \in\{1,2, \ldots, n\}$ which form a strict 3-cycle $\left(v_{i},\left(v_{i}, v_{j}\right), v_{j},\left(v_{j}, v_{k}\right), v_{k},\left(v_{k}, v_{i}\right), v_{i}\right)$. The cycle is strict, and thus, there is at least one arc whose weight is greater than $s_{0}$.

(1) If all the three weights are greater than $s_{0}$, it is obvious that $P$ is not weakly consistent.

(2) If two of the three weights are greater than $s_{0}$, we denote $w\left(v_{i}, v_{j}\right) \succ s_{0}, w\left(v_{j}, v_{k}\right) \succ s_{0}$, and $w\left(v_{k}, v_{i}\right) \simeq s_{0}$. We have $l_{j k} \succ s_{0}$ (i.e., $\left.l_{j k} \succeq s_{0}\right)$ and $l_{k i} \simeq s_{0}$ (i.e., $l_{k i} \succeq s_{0}$ ), but $l_{j i} \prec s_{0}$. Thus, $P$ is not weakly consistent.

(3) If one of the three weights is greater than $s_{0}$, we denote $w\left(v_{i}, v_{j}\right) \succ s_{0}, w\left(v_{j}, v_{k}\right) \simeq s_{0}$, and $w\left(v_{k}, v_{i}\right) \simeq s_{0}$. Accordingly, $l_{j k} \succeq s_{0}$ and $l_{k i} \succeq s_{0}$. However, $l_{j i} \prec s_{0}$. Thus, $P$ is not weakly consistent.

Theorem 6 ensures that the weak consistency can be identified by checking the existence of strict 3-cycle. In order to identify whether a 3-cycle is strict or not, we need to find out the walk of a cycle at first.

Algorithm 1: Input: a LHPR $P=\left(l_{i j}\right)_{n \times n}$. Output: $P$ is weakly consistent or not.

Step 1: Construct the adjacent matrix $M$ according to Eq.(15); let $c=1$.

Step 2: For any $i, j, k=1,2, \ldots, n$, if $M_{i j} M_{j k} M_{k i}=1$, then let $l_{\max }=\max \left\{l_{i j}, l_{j k}, l_{k i}\right\}$ : if $l_{\max } \succ s_{0}$, then $c=0$, break; else, continue.

Step 3: If $c=1$, then $P$ is weakly consistent; else $P$ is not.

Note that, different from the similar weak consistency identification algorithms [28], [33], the above algorithm checks the P-graph of $P$ based on two levels. First, $M_{i j} M_{j k} M_{k i}=1$ is to identify the existence of a 3-cycle. In addition, if $l_{\max } \succ s_{0}$, then the found 3-cycle is a strict 3-cycle. The algorithm is terminated if a strict 3-cycle is found or $i, j, k$ have gone through $1,2, \ldots, n$. Moreover, for a preference relation, $n$ is usually suggested to be less than 9 [34]. Thus, the complexity of Algorithm 1 is acceptable.

Example 3: Consider the LHPR $P$ in Example 2, according to Algorithm 1, there is no 3-cycle in the P-graph, then $P$ is weakly consistent. 


\section{Additive consistency of LHPRs}

Below we consider how to identify whether a LHPR is additively consistent or not.

Theorem 7: If a LHPR $P=\left(l_{i j}\right)_{n \times n}$ is reciprocal, then the following statements are equivalent:

(1) $l_{i j} \oplus l_{j k} \simeq l_{i k}, \forall i, j, k$;

(2) $l_{i j} \oplus l_{j k} \simeq l_{i k}, \forall i<j<k$;

(3) $l_{i, i+1} \oplus l_{i+1, i+2} \oplus \cdots \oplus l_{j-1, j} \oplus l_{j, i} \simeq s_{0}, \forall i<j$.

Proof. (1) $\Rightarrow(2)$ : It's obvious.

(2) $\Rightarrow(1)$ : Let us consider the order of $i, j, k$. If $i=j=k$, $l_{i j} \oplus l_{j k}=s_{0}=l_{i k}$; if two of the three are equal, say, if $i=j \neq k, l_{i j} \oplus l_{j k}=s_{0} \oplus l_{i k}=l_{i k}$. Then we consider the case $i \neq j \neq k$ :

(i) If $i<j<k$, then apparently $l_{i j} \oplus l_{j k} \simeq l_{i k}$ according to (2);

(ii) If $i<k<j$, then we have $l_{i k} \oplus l_{k j} \simeq l_{i j}$. Thus,

$l_{i j} \oplus l_{j k}=\left(l_{i k} \oplus l_{k j}\right) \oplus l_{j k}=l_{i k} \oplus\left(l_{k j} \oplus l_{j k}\right) \simeq l_{i k} \oplus s_{0}=l_{i k}$

(iii) Similar to (ii), $l_{i j} \oplus l_{j k} \simeq l_{i k}$ holds if $j<i<k$, $j<k<i, k<i<j$, and $k<j<i$.

Thus for any $i, j, k=1,2, \ldots, n, l_{i j} \oplus l_{j k} \simeq l_{i k}$.

(2) $\Rightarrow(3)$ : Let $j=i+1$ and $k=i+2$ in (2), we get

$$
l_{i, i+1} \oplus l_{i+1, i+2} \simeq l_{i, i+2}
$$

Similarly, we can get a collection of equations:

$$
l_{i, i+2} \oplus l_{i+2, i+3} \simeq l_{i, i+3}, \ldots, l_{i, j-1} \oplus l_{j-1, j} \simeq l_{i j}
$$

. Moreover, $l_{i j} \oplus l_{j, i} \simeq s_{0}$. The accumulation of these equations results in:

$$
l_{i, i+1} \oplus l_{i+1, i+2} \oplus \cdots \oplus l_{j-1, j} \oplus l_{j, i} \simeq s_{0}
$$

(3) $\Rightarrow(2)$ : Let $i<j<k$, then we obtain

$$
\begin{gathered}
l_{i, i+1} \oplus l_{i+1, i+2} \oplus \cdots \oplus l_{j-1, j} \oplus l_{j i} \simeq s_{0} \\
l_{j, j+1} \oplus l_{j+1, j+2} \oplus \cdots \oplus l_{k-1, k} \oplus l_{k j} \simeq s_{0}
\end{gathered}
$$

and

$$
l_{i, i+1} \oplus l_{i+1, i+2} \oplus \cdots \oplus l_{k-1, k} \oplus l_{k i} \simeq s_{0}
$$

from (3). Accumulating the first two, we get

$$
l_{i, i+1} \oplus l_{i+1, i+2} \oplus \cdots \oplus l_{k-1, k} \oplus l_{j i} \oplus l_{k j} \simeq s_{0}
$$

Comparing it with the third equation, it is obvious that $l_{j i} \oplus$ $l_{k j} \simeq l_{k i}$, which also means

$$
\left(s_{0} \ominus l_{i j}\right) \oplus\left(s_{0} \ominus l_{j k}\right) \simeq\left(s_{0} \ominus l_{i k}\right)
$$

According to Theorem 2, $l_{i j} \oplus l_{j k} \simeq l_{i k}$.

In Theorem 7, (2) presents a simple way to check the additive consistency of a LHPR. In fact, it is enough to check the values of $l_{i j}, l_{j k}$, and $l_{i k}$ having $i \leq j \leq k$ for a given reciprocal LHPR. Besides, (3) enables to generate an additively consistent LHPR by means of $n-1$ entries: $l_{12}, l_{23}, \ldots, l_{n-1, n}$.
1) Constructing an additively consistent LHPR from n-1 entries: Incomplete preference relations are frequently considered to facilitate experts providing their preference information. Ideally, if each object is compared by only one time, then we have $n-1$ known entries of the preference relation. An additively consistent preference relation can always be generated in this case.

The algorithm can be depicted by two phases: computation and normalization. The first phase (Steps 1-2) computes all the missing entries, and then the second phase (Steps 3-4) normalizes the results so that they could be interpretable in the original domain. Specifically,

Algorithm 2: Input: An incomplete LHPR $P=\left(l_{i j}\right)_{n \times n}$ with known entries $l_{12}, l_{23}, \ldots, l_{n-1, n} \in \overline{\mathcal{L}}$. Output: A completed additively consistent LHPR $P$.

Step 1: For any $i<j$, if $l_{i j} \notin\left\{l_{12}, l_{23}, \ldots, l_{n-1, n}\right\}$, do

$$
l_{i j}=l_{i, i+1} \oplus l_{i+1, i+2} \oplus \cdots \oplus l_{j-1, j}
$$

Step 2: For any $i=j, l_{i i}=s_{0}$; for any $i>j, l_{i j}=$ $N e g\left(l_{j i}\right)$.

Step 3: Find an entry $l_{i_{0}, j_{0}}=\left\langle h_{t_{i_{0}, j_{0}}}, s_{\alpha_{i_{0}, j_{0}}}\right\rangle$ satisfying $l_{i_{0}, j_{0}} \succeq l_{i j}$, for any $i, j=1,2, \ldots, n$. If $s_{\alpha_{i_{0}, j_{0}}} \leq s_{\tau}$, then go to Step 5; else, go to Step 4.

Step 4: A new LHPR $P^{\prime}=f(P)$ where the function

$$
f:\left[s_{-\alpha_{i_{0}, j_{0}}}, s_{\alpha_{i_{0}, j_{0}}}\right] \rightarrow\left[s_{-\tau}, s_{\tau}\right]
$$

normalizes the second component of each LTWH in $P$, such that $f\left(s_{\alpha}\right)=s_{\beta}$ with $\beta=\tau \alpha / \alpha_{i_{0}, j_{0}}$. Let $P=P^{\prime}$.

Step 5: End.

Theorem 8: Algorithm 2 outputs an additively consistent LHPR.

Proof. Given $l_{12}, l_{23}, \ldots, l_{n-1, n} \in \overline{\mathcal{L}}$, it is apparent that the computational results of Step 2 satisfy $l_{i j} \oplus l_{j k} \simeq l_{i k}$, $\forall i, j, k$, according to Theorem 7. Thus, if $s_{\alpha_{i_{0}, j_{0}}} \leq s_{\tau}$, then the resultant $P$ is additively consistent; else we have to clarify that $f$ mapping $P$ to an additively consistent LHPR whose entries are in $\overline{\mathcal{L}}$.

Denote $P=\left(l_{i j}\right)_{n \times n}=\left(\left\langle h_{t_{i j}}, s_{\alpha_{i j}}\right\rangle\right)_{n \times n}, P^{\prime}=\left(l_{i j}^{\prime}\right)_{n \times n}$ $=\left(\left\langle h_{t_{i j}}, s_{\alpha_{i j}}^{\prime}\right\rangle\right)_{n \times n}$.

(1) Obviously, $f\left(s_{-\alpha_{i_{0}, j_{0}}}\right)=s_{-\tau}, f\left(s_{\alpha_{i_{0}, j_{0}}}\right)=s_{\tau}$. Thus, the entries are normalized into the range $\overline{\mathcal{L}}$.

(2) For any $i, j=1,2, \ldots, n$,

$$
\left\langle h_{t_{i j}}, s_{\alpha_{i j}}^{\prime}\right\rangle \oplus\left\langle h_{t_{j i}}, s_{\alpha_{j i}}^{\prime}\right\rangle=\left\langle h_{t_{i j}}, f\left(s_{\alpha_{i j}}\right)\right\rangle \oplus\left\langle h_{t_{j i}}, f\left(s_{\alpha_{j i}}\right)\right\rangle
$$

where

$$
f\left(s_{\alpha_{i j}}\right) \oplus f\left(s_{\alpha_{j i}}\right)=\left(\tau / \alpha_{i_{0}, j_{0}}\right) s_{\alpha_{i j}} \oplus s_{\alpha_{j i}}=\left(\tau / \alpha_{i_{0}, j_{0}}\right) s_{0}
$$

according to Step 2. Thus, $\left\langle h_{t_{i j}}, s_{\alpha_{i j}}^{\prime}\right\rangle \oplus\left\langle h_{t_{j i}}, s_{\alpha_{j i}}^{\prime}\right\rangle \simeq s_{0}$. The reciprocal property is proven.

(3) For any $i, j, k=1,2, \ldots, n$,

$$
\begin{aligned}
l_{i j}^{\prime} \oplus l_{j k}^{\prime} & =\left\langle h_{t_{i j}}, s_{\alpha_{i j}}^{\prime}\right\rangle \oplus\left\langle h_{t_{j k}}, s_{\alpha_{j k}}^{\prime}\right\rangle \\
& =\left\langle h_{\max \left\{t_{i j}, t_{j k}\right\}}, f\left(s_{\alpha_{i j}}\right) \oplus f\left(s_{\alpha_{j k}}\right)\right\rangle
\end{aligned}
$$

where

$$
\begin{aligned}
f\left(s_{\alpha_{i j}}\right) \oplus f\left(s_{\alpha_{j k}}\right) & =\left(\tau / \alpha_{i_{0}, j_{0}}\right) s_{\alpha_{i j}} \oplus s_{\alpha_{j k}} \\
& =\left(\tau / \alpha_{i_{0}, j_{0}}\right) s_{\alpha_{i k}}=f\left(s_{\alpha_{i k}}\right)
\end{aligned}
$$


according to Step 1 and Theorem 7. Thus, $l_{i j}^{\prime} \oplus l_{j k}^{\prime}=$ $\left\langle h_{\max \left\{t_{i j}, t_{j k}\right\}}, s_{\alpha_{i k}}^{\prime}\right\rangle \simeq l_{i k}^{\prime}$. The additive consistency is proven.

Note that, due to the basic operations in Definition 6, the uncertainties of the known entries which are expressed by weakened hedges, will be transferred to the missing entries during the generating process.

Example 4: Given an incomplete LHPR $P=\left(l_{i j}\right)_{4 \times 4}$ with three known entries: $l_{12}=s_{1}, l_{23}=\left\langle h_{1}, s_{2}\right\rangle, l_{34}=\left\langle h_{2}, s_{-1}\right\rangle$, based on the LTS $S$ and the WHS $\bar{H}$ defined in Example 1. According to Steps 1-2 of Algorithm 2, P can be completed as:

$$
P=\left(\begin{array}{cccc}
s_{0} & s_{1} & \left\langle h_{1}, s_{3}\right\rangle & \left\langle h_{2}, s_{2}\right\rangle \\
s_{-1} & s_{0} & \left\langle h_{1}, s_{2}\right\rangle & \left\langle h_{2}, s_{1}\right\rangle \\
\left\langle h_{1}, s_{-3}\right\rangle & \left\langle h_{1}, s_{-2}\right\rangle & s_{0} & \left\langle h_{2}, s_{-1}\right\rangle \\
\left\langle h_{2}, s_{-2}\right\rangle & \left\langle h_{2}, s_{-1}\right\rangle & \left\langle h_{2}, s_{1}\right\rangle & s_{0}
\end{array}\right)
$$

where, for example, $l_{14}=l_{12} \oplus l_{23} \oplus l_{34}=s_{1} \oplus\left\langle h_{1}, s_{2}\right\rangle \oplus$ $\left\langle h_{2}, s_{-1}\right\rangle=\left\langle h_{2}, s_{2}\right\rangle, l_{41}=N e g\left(l_{14}\right)=\left\langle h_{2}, s_{-2}\right\rangle$. We can see that the greatest element is $l_{13}=\left\langle h_{1}, s_{3}\right\rangle$. Because $s_{3} \prec$ $s_{\tau}=s_{4}$, the algorithm is terminated.

2) Constructing an additively consistent LHPR by a reciprocal LHPR: Besides, an additively consistent LHPR can be generated based on a given LHPR. Consider the SPgraph of a LHPR $P$, if $P$ is additively consistent, then given $i, j=1,2, \ldots, n, w\left(v_{i}, v_{j}\right)$ should be equivalent to the fuzzy length of path $\left(v_{i},\left(v_{i}, v_{k}\right), v_{k},\left(k_{i}, v_{j}\right), v_{j}\right)$, for any $k=1,2, \ldots, n$. This fact motivates us to estimate $l_{i j}$ the set of paths which are from $v_{i}$ to $v_{j}$ through any intermediate vertices. Formally, we have

Theorem 9: Given a reciprocal LHPR $P=\left(l_{i j}\right)_{n \times n}$, let $\bar{P}=\left(\bar{l}_{i j}\right)_{n \times n}$ with

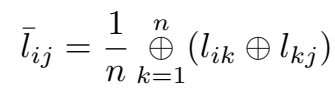

where $i, j=1,2, \ldots, n$, then $\bar{P}$ is additively consistent.

Proof. (1) For any $i, j=1,2, \ldots, n$,

$$
\begin{aligned}
\bar{l}_{j i} & =\frac{1}{n}{\underset{k=1}{n}}_{k}^{n}\left(l_{j k} \oplus l_{k i}\right)=\frac{1}{n} \underset{k=1}{\oplus}\left(N e g\left(l_{k j}\right) \oplus \operatorname{Neg}\left(l_{i k}\right)\right) \\
& =\frac{1}{n} \operatorname{Neg}\left(\bigoplus_{k=1}^{n}\left(l_{i k} \oplus l_{k j}\right)\right)=\operatorname{Neg}\left(\bar{l}_{i j}\right),
\end{aligned}
$$

thus $\bar{P}$ is reciprocal.

(2) For any $i, j, k=1,2, \ldots, n$,

$$
\begin{aligned}
& \bar{l}_{i k} \oplus \bar{l}_{k j}=\frac{1}{n} \underset{k_{1}=1}{\oplus}\left(l_{i, k_{1}} \oplus l_{k_{1}, k}\right) \oplus \frac{1}{n}{\stackrel{\oplus}{k_{2}=1}}_{n}^{n}\left(l_{k, k_{2}} \oplus l_{k_{2}, j}\right) \\
& =\frac{1}{n} \underset{k_{1}=1}{\oplus}\left(l_{i, k_{1}} \oplus l_{k_{1}, k} \oplus l_{k, k_{1}} \oplus l_{k_{1}, j}\right) \\
& =\frac{1}{n} \underset{k_{1}=1}{\oplus}\left(l_{i, k_{1}} \oplus l_{k_{1}, j}\right)=\bar{l}_{i j}
\end{aligned}
$$

thus $\bar{P}$ is additively consistent.

In practice, it is not very interesting to generate such a LHPR based on arbitrary reciprocal LHPR. However, the generation process implies another way to check if a LHPR is additively consistent.
Theorem 10: Let $P=\left(l_{i j}\right)_{n \times n}$ be a reciprocal LHPR and $\bar{P}=\left(\bar{l}_{i j}\right)_{n \times n}$ be the LHPR generated by Eq.(16). $P$ is additively consistent if and only if $P \simeq \bar{P}$.

Proof. $(\Leftarrow)$ : If $P \simeq \bar{P}$, according to Theorem $9, \bar{P}$ is additively consistent. For any $i, j, k=1,2, \ldots, n, l_{i k} \oplus l_{k j} \simeq$ $\bar{l}_{i k} \oplus \bar{l}_{k j} \simeq \bar{l}_{i j} \simeq l_{i j}$, then $P$ is also additively consistent.

$(\Rightarrow)$ : If $P$ is additively consistent, then $l_{i k} \oplus l_{k j} \simeq l_{i j}$ for any $i, j, k=1,2, \ldots, n$. Accordingly,

$$
\bar{l}_{i j}=\frac{1}{n} \bigoplus_{k=1}^{n}\left(l_{i k} \oplus l_{k j}\right) \simeq \frac{1}{n} \bigoplus_{k=1}^{n} l_{i j}=l_{i j}
$$

for any $i, j=1,2, \ldots, n$. Thus $P \simeq \bar{P}$.

According to Theorem 10, an additively consistent LHPR should be equivalent to the LHPR generated by Eq.(16).

Example 5: We have proven that the LHPR in Example 2 is weakly consistent. According to Eq.(16), a new LHPR $\bar{P}=$ $\left(\bar{l}_{i j}\right)_{5 \times 5}$ can be generated. For instance, $\bar{l}_{12}=\frac{1}{5} \underset{k=1}{\oplus}\left(l_{1 k} \oplus\right.$ $\left.l_{k 2}\right)=\frac{1}{5}\left(s_{0} \oplus\left\langle h_{1}, s_{-2}\right\rangle\right) \oplus\left(\left\langle h_{1}, s_{-2}\right\rangle \oplus s_{0}\right) \oplus\left(\left\langle h_{1}, s_{-3}\right\rangle \oplus\right.$ $\left.s_{-2}\right) \oplus\left(s_{-1} \oplus\left\langle h_{1}, s_{-1}\right\rangle\right) \oplus\left(\left\langle h_{2}, s_{2}\right\rangle \oplus s_{-2}\right)=\left\langle h_{2}, s_{-2.2}\right\rangle$. Moreover, we have:

$$
\bar{P}=\left(\begin{array}{ccc}
\left\langle h_{2}, s_{0}\right\rangle & \left\langle h_{2}, s_{-2.2}\right\rangle & \left\langle h_{2}, s_{-1.8}\right\rangle \\
\left\langle h_{2}, s_{2.2}\right\rangle & \left\langle h_{1}, s_{0}\right\rangle & \left\langle h_{1}, s_{0.4}\right\rangle \\
\left\langle h_{2}, s_{1.8}\right\rangle & \left\langle h_{1}, s_{-0.4}\right\rangle & \left\langle h_{1}, s_{0}\right\rangle \\
\left\langle h_{2}, s_{0.6}\right\rangle & \left\langle h_{1}, s_{-1.6}\right\rangle & \left\langle h_{1}, s_{-1.2}\right\rangle \\
\left\langle h_{2}, s_{-0.6}\right\rangle & \left\langle h_{2}, s_{-2.8}\right\rangle & \left\langle h_{2}, s_{-2.4}\right\rangle \\
\left\langle h_{2}, s_{-0.6}\right\rangle & \left\langle h_{2}, s_{0.6}\right\rangle \\
\left\langle h_{1}, s_{1.6}\right\rangle & \left\langle h_{2}, s_{2.8}\right\rangle \\
\left\langle h_{1}, s_{1.2}\right\rangle & \left\langle h_{2}, s_{2.4}\right\rangle \\
\left\langle h_{1}, s_{0}\right\rangle & \left\langle h_{2}, s_{1.2}\right\rangle \\
\left\langle h_{2}, s_{-1.2}\right\rangle & \left\langle h_{2}, s_{0}\right\rangle
\end{array}\right)
$$

Obviously, $P \simeq \bar{P}$ is not true, thus $P$ is not additively consistent.

If a LHPR is not additively consistent, then the degree of additive consistency could be measured by the similarity or deviation between $P$ and $\bar{P}$. This will be presented in detail in the next section.

\section{CONSISTENCY IMPROVING OF LHPRS}

The additive consistency is an ideal case of preference relation, which might be hard to reach. The weak consistency often serves as the boundary condition that a preference relation should satisfy. In applications, the degree of additive consistency is frequently considered. If a given LHPR approaches to its additively consistent version, then it would be satisfactory and acceptable by a decision-maker. The deviation between two LHPRs, which is vital to quantify the degree of consistency, is defined based on the deviation between each pair of upper diagonal elements in the LTWHs:

Definition 13: Given two reciprocal LHPRs $P=\left(l_{i j}\right)_{n \times n}$ and $P^{\prime}=\left(l_{i j}^{\prime}\right)_{n \times n}$, their deviation is defined as:

$$
d\left(P, P^{\prime}\right)=\frac{2}{n(n-1)} \underset{i<j}{\oplus} d\left(l_{i j}, l_{i j}^{\prime}\right)
$$

Definition 14 can be regarded as a tool of consistency improving. Firstly, we will employ it to improve a LHPR to satisfy the weak transitivity. 


\section{A. Improving a LHPR to satisfy weak consistency}

It is generally required that a LHPR is weakly consistent. If not, an approach should be developed to improve the consistency of the LHPR. Interactive approaches, such as the idea in [32], detect the illogical arcs in the graph and receive feedbacks from experts. Automatic approaches, like the idea in [28], [33], improves the preference relation based on the original information and some specific revision rules. In the sequel, we propose an automatic approach to improve a LHPR which is not weakly consistent.

Algorithm 3: Input: a reciprocal LHPR $P, \lambda \in(0,1)$; Output: a weakly consistent LHPR $P^{(m)}$.

Step 1: Let $P^{(0)}=P, m=0$.

Step 2: According to Algorithm 1, if $P^{(m)}$ is weakly consistent, go to Step 4; else, generate $\bar{P}^{(m)}$ by using Eq.(16), go to Step 3.

Step 3: Let $P^{(m+1)}=(1-\lambda) P^{(m)} \oplus \lambda \bar{P}^{(m)}, m=m+1$. Go to Step 2.

Step 4: Output $P^{(m)}$.

The iterative algorithm revises the entries of a given LHPR automatically and maintains the original opinions as much as possible. To this end, $\lambda$ plays an important role. In fact, $\lambda$ implies to what extent the original information should be maintained. If $\lambda=0$, then only the original information in $P$ is considered; if $\lambda=1$, then the original information is completely ignored.

In Algorithm 3, the additively consistent LHPR $\bar{P}^{(m)}$ is generated in each iteration. This is rational but results in the complexity of the algorithm. To improve Algorithm 3, we present the following theorem at first:

Theorem 11: Given any $\bar{P}^{(m)}$ and $\bar{P}^{(m+1)}$ derived in Algorithm 3, $m \geq 0, \lambda \in(0,1)$, we have $\bar{P}^{(m)} \simeq \bar{P}^{(m+1)}$.

Proof. Denote $P^{(m)}=\left(l_{i j}^{(m)}\right)_{n \times n}=\left(\left\langle h_{t_{i j}^{(m)}}, s_{\alpha_{i j}^{(m)}}\right\rangle\right)_{n \times n}$, $\bar{P}^{(m)}=\left(\bar{l}^{(m)}\right)_{n \times n}$.

(1) Firstly, we have to prove that $P^{(m+1)}$ is a reciprocal LHPR. In fact,

$$
\begin{aligned}
l_{i j}^{(m+1)}= & (1-\lambda) l_{i j}^{(m)} \oplus \lambda \frac{1}{n} \underset{k=1}{\oplus}\left(l_{i k}^{(m)} \oplus l_{k j}^{(m)}\right) \\
= & \left\langle h_{t_{i j}^{(m)}}, s_{(1-\lambda) \alpha_{i j}^{(m)}}\right\rangle \\
& \oplus \lambda \frac{1}{n} \underset{k=1}{\oplus}\left\langle h_{\max \left\{t_{i k}^{(m)}, t_{k j}^{(m)}\right\}}, s_{\alpha_{i k}^{(m)}+\alpha_{k j}^{(m)}}\right\rangle \\
= & \left\langle h_{t_{i j}^{(m)}}, s_{(1-\lambda) \alpha_{i j}^{(m)}}\right\rangle\left\langle h_{t_{0}^{(i j)}}, s_{\lambda \alpha_{0}^{(i j)}}\right\rangle,
\end{aligned}
$$

where $t_{0}^{(i j)}=\max _{k}\left\{\max \left\{t_{i k}^{(m)}, t_{k j}^{(m)}\right\}\right\}, \quad \alpha_{0}^{(i j)}=$ $\frac{1}{n} \sum_{k=1}^{n}\left(\alpha_{i k}^{(m)}+\alpha_{k j}^{(m)}\right)$. Then

$$
l_{i j}^{(m+1)}=\left\langle h_{\max \left\{t_{i j}^{(m)}, t_{0}^{(i j)}\right\}}, s_{(1-\lambda) \alpha_{i j}^{(m)}+\lambda \alpha_{0}^{(i j)}}\right\rangle
$$

It is clear that $h_{\max \left\{t_{i j}^{(m)}, t_{0}^{(i j)}\right\}} \in \bar{H}, s_{(1-\lambda) \alpha_{i j}^{(m)}+\lambda \alpha_{0}^{(i j)}} \in \bar{S}$, thus $l_{i j}^{(m+1)} \in \overline{\mathcal{L}}$ and $P^{(m+1)}$ is a LHPR. Furthermore, if $m=0$, then $P$ is reciprocal. Assume that $P^{(m)}$ is reciprocal, which means $l_{i j}^{(m)}=N e g\left(l_{j i}^{(m)}\right)$ for any $i, j=1,2, \ldots, n$. Then

$$
\begin{aligned}
l_{j i}^{(m+1)} & =\left\langle h_{\max \left\{t_{j i}^{(m)}, t_{0}^{(j i)}\right\}}, s_{\left.(1-\lambda) \alpha_{j i}^{(m)}+\lambda \alpha_{0}^{(j i)}\right\rangle}\right\rangle \\
& =\left\langle h_{\max \left\{t_{i j}^{(m)}, t_{0}^{(i j)}\right\}}, s_{-\left((1-\lambda) \alpha_{i j}^{(m)}+\lambda \alpha_{0}^{(i j)}\right)}\right\rangle \\
& =N \operatorname{eg}\left(l_{i j}^{(m+1)}\right)
\end{aligned}
$$

thus $P^{(m+1)}$ is reciprocal.

(2) We prove $\bar{P}^{(m)} \simeq \bar{P}^{(m+1)}$. According to $l_{i j}^{(m+1)}$ derived in (1), we have:

$$
\begin{aligned}
& \bar{l}_{i j}^{(m+1)}=\frac{1}{n} \bigoplus_{k=1}^{n}\left(\left\langle h_{t_{0}^{(i k)}}, s_{\alpha_{0}^{(i k)}}\right\rangle \oplus\left\langle h_{t_{0}^{(k j)}}, s_{\alpha_{0}^{(k j)}}\right\rangle\right) \\
& =\frac{1}{n} \bigoplus_{k=1}^{n}\left\langle h_{\max \left\{t_{0}^{(i k)}, t_{0}^{(k j)}\right\}}, s_{\left.\alpha_{0}^{(i k)}+\alpha_{0}^{(k j)}\right\rangle}\right\rangle \\
& =\left\langle h_{t_{1}^{(i j)}}, s_{\alpha_{1}^{(i j)}}\right\rangle \text {, }
\end{aligned}
$$

where $t_{1}^{(i j)}=\max _{k}\left\{\max \left\{t_{0}^{(i k)}, t_{0}^{(k j)}\right\}\right\}$ and $\alpha_{1}^{(i j)}=$ $\frac{1}{n} \sum_{k=1}^{n}\left(\alpha_{0}^{(i k)}+\alpha_{0}^{(k j)}\right)$. Especially,

$$
\begin{aligned}
\alpha_{1}^{(i j)}= & \frac{1}{n} \sum_{k=1}^{n}\left(\frac{1}{n} \sum_{k_{1}=1}^{n}\left(\alpha_{i k_{1}}^{(m)}+\alpha_{k_{1} k}^{(m)}\right)\right. \\
& \left.\quad+\frac{1}{n} \sum_{k_{2}=1}^{n}\left(\alpha_{k k_{2}}^{(m)}+\alpha_{k_{2} j}^{(m)}\right)\right) \\
= & \frac{1}{n^{2}} \sum_{k=1}^{n} \sum_{k_{1}=1}^{n}\left(\alpha_{i k_{1}}^{(m)}+\alpha_{k_{1} k}^{(m)}+\alpha_{k k_{1}}^{(m)}+\alpha_{k_{1} j}^{(m)}\right) \\
= & \frac{1}{n^{2}} \sum_{k=1}^{n} \sum_{k_{1}=1}^{n}\left(\alpha_{i k_{1}}^{(m)}+\alpha_{k_{1} j}^{(m)}\right) \\
= & \frac{1}{n} \sum_{k_{1}=1}^{n}\left(\alpha_{i k_{1}}^{(m)}+\alpha_{k_{1} j}^{(m)}\right)=\alpha_{0}^{(i j)},
\end{aligned}
$$

which means $\bar{l}_{i j}^{(m+1)} \simeq \bar{l}_{i j}^{(m)}$. Therefore, $\bar{P}^{(m)} \simeq \bar{P}^{(m+1)}$.

Thus, according to (3) of Theorem 3 and Theorem 11, it is not necessary to generate $\bar{P}^{(m)}$ in each loop of Algorithm 3 . The algorithm can be improved to the following version:

Algorithm 4: Input: a reciprocal LHPR $P, \lambda \in(0,1)$; Output: a weakly consistent LHPR $P^{(m)}$.

Step 1: Let $P^{(0)}=P, m=0$, generate $\bar{P}$ by using Eq.(16).

Step 2: According to Algorithm 1, if $P^{(m)}$ is weakly consistent, then go to Step 4; else, go to Step 3.

Step 3: Let $P^{(m+1)}=(1-\lambda) P^{(m)} \oplus \lambda \bar{P}, m=m+1$. Go to Step 2.

Step 4: Output $P^{(m)}$.

The convergence of Algorithm 4 can be identified as follows:

Theorem 12: Algorithm 4 outputs a weakly consistent LHPR within finite loops.

Proof. Let $P^{(m)}=\left(l_{i j}^{(m)}\right)_{n \times n}$ and $\bar{P}=\left(\bar{l}_{i j}\right)_{n \times n}$ which is generated from $P$. It is sufficient if the deviation between 
$P^{(m)}$ and $\bar{P}$ reduces in each loop. In fact, according to the proof of Theorem 11, we have

$$
\begin{aligned}
d\left(l_{i j}^{(m+1)}, \bar{l}_{i j}\right)= & d\left(\left\langleh_{\max \left\{t_{i j}^{(m)}, t_{0}^{(i j)}\right\}}, s_{\left.(1-\lambda) \alpha_{i j}^{(m)}+\lambda \alpha_{0}^{(i j)}\right\rangle,}\right.\right. \\
& \left.\left\langle h_{t_{0}^{(i j)}}, s_{\alpha_{0}^{(i j)}}\right\rangle\right) \\
= & \left\langle h_{\max \left\{t_{i j}^{(m)}, t_{0}^{(i j)}\right\}}, s_{\left|(1-\lambda) \alpha_{i j}^{(m)}+\lambda \alpha_{0}^{(i j)}-\alpha_{0}^{(i j)}\right|}\right\rangle \\
= & \left\langle h_{\max \left\{t_{i j}^{(m)}, t_{0}^{(i j)}\right\}}, s_{\mid(1-\lambda)\left(\alpha_{i j}^{(m)}-\alpha_{0}^{(i j)} \mid\right.}\right\rangle \\
\simeq & (1-\lambda)\left\langle h_{\max \left\{t_{i j}^{(m)}, t_{0}^{(i j)}\right\}}, s_{\mid\left(\alpha_{i j}^{(m)}-\alpha_{0}^{(i j)} \mid\right.}\right\rangle \\
= & (1-\lambda) d\left(l_{i j}^{(m)}, \bar{l}_{i j}\right) \prec d\left(l_{i j}^{(m)}, \bar{l}_{i j}\right) .
\end{aligned}
$$

Thus $d\left(P^{(m+1)}, \bar{P}\right) \prec d\left(P^{(m)}, \bar{P}\right)$. If $m \rightarrow+\infty$, then $P^{(m)}$ approaches an additively consistent LHPR. According to (2) of Theorem 4, the proof is completed.

\section{B. Improving the degree of addive consistency}

Obviously, the above algorithms improve a LHPR by letting it approach to an additively consistent LHPR. In applications, if weak consistency does not meet the requirement of a decision-maker, then the concept of the degree of additive consistency can be introduced so that the decision-maker can express the threshold of satisfactory consistency. Given $P$, the degree of additive consistency of a LHPR can be measured by the deviation between each entry in the upper triangular position $l_{i j}(i<j)$ and its ideal value.

Definition 14: The degree of additive consistency of $P$ is defined as:

$$
D(P)=s_{\tau} \ominus d(P, \bar{P})
$$

where $\bar{P}$ is the LHPR generated by Eq.(16) based on $P$.

The larger $D(P)$ implies the larger degree of additive consistency of $P$. Linguistically, take the LTS in Example 1 for instance, if $D(P) \simeq s_{4}$, then we can assert that the degree of additive consistency of $P$ is "extremely good". Moreover, we have the following theorem:

Theorem 13: For any reciprocal LHPR $P=\left(l_{i j}\right)_{n \times n}$, $D(P) \simeq s_{\tau}$ if and only if $P$ is additively consistent.

Proof. Let $\bar{P}=\left(\bar{l}_{i j}\right)_{n \times n}$ represent the additively consistent LHPR generated by Eq.(16).

$(\Rightarrow)$ According to Definition 7, $d\left(l_{1}, l_{2}\right) \succeq s_{0}$ for any $l_{1}, l_{2} \in \overline{\mathcal{L}}$. Thus $D(P) \simeq s_{\tau}$ means $d\left(l_{i j}, \bar{l}_{i j}\right) \simeq s_{0}$ for any $i<j$ and $i, j=1,2, \ldots, n$. Moreover, $P$ is reciprocal, thus $l_{i j} \simeq \bar{l}_{i j}$ for any $i, j=1,2, \ldots, n$. Because $\bar{P}$ is additively consistent, $P$ is also additively consistent.

$(\Leftarrow)$ If $P$ is additively consistent, then according to Theorem $10, P \simeq \bar{P}$. For any $i, j=1,2, \ldots, n, l_{i j} \simeq \bar{l}_{i j}, d\left(l_{i j}, \bar{l}_{i j}\right) \simeq$ $s_{0}$, which means $d(P, \bar{P}) \simeq s_{0}$. Thus $D(P) \simeq s_{\tau}$.

In application, it may be acceptable if the degree of additive consistency of a LHPR is big enough. Given a threshold $\delta$ determined by the decision-maker, the concept of satisfactory consistency is defined below:

Definition 15: A LHPR $P$ is said to be of satisfactory consistency if $D(P) \geq \delta$, where $\delta \in\left\{s_{1}, s_{2}, \ldots, s_{\tau}\right\}$.

Given a LHPR, the satisfactory consistency can be achieved automatically by slightly revise Algorithm 4, which is depicted as follows:
Algorithm 5: Input: a reciprocal LHPR $P, \lambda \in(0,1), \delta$; Output: a LHPR $P^{(m)}$ with satisfactory consistency.

Step 1: Let $P^{(0)}=P, m=0$, generate $\bar{P}$ by using Eq.(16).

Step 2: Compute $D\left(P^{(m)}\right)$ by using Eq.(18). If $D\left(P^{(m)}\right) \succeq$ $\delta$, go to Step 4; else, go to Step 3.

Step 3: Let $P^{(m+1)}=(1-\lambda) P^{(m)} \oplus \lambda \bar{P}, m=m+1$. Go to Step 2.

Step 4: Output $P^{(m)}$.

According to the proof of Theorem 12, we have

$$
\begin{aligned}
d\left(P^{(m+1)}, \bar{P}\right) & =\frac{2}{n(n-1)} \bigoplus_{i<j} d\left(l_{i j}^{(m+1)}, \bar{l}_{i j}\right) \\
& \simeq \frac{2}{n(n-1)} \bigoplus_{i<j}(1-\lambda) d\left(l_{i j}^{(m)}, \bar{l}_{i j}\right) \\
& =(1-\lambda) d\left(P^{(m)}, \bar{P}\right) .
\end{aligned}
$$

Assume that $d(P, \bar{P}) \simeq s_{\alpha}$, i.e., $D(P) \simeq s_{\tau-\alpha}$. Then we have $D\left(P^{(m)}\right) \simeq s_{\tau-(1-\lambda)^{m} \alpha}$. If $\delta=s_{\beta}(\beta>\alpha)$, then Algorithm 5 will be terminated once $\tau-(1-\lambda)^{m} \alpha \geq \beta$, i.e., $m \geq$ $\log _{1-\lambda} \frac{\tau-\beta}{\alpha}$.

Example 6: Continue with the LHPR $P$ in Example 2. According to Eqs.(17-18), $D(P)=\left\langle h_{2}, s_{2.98}\right\rangle$. Assume that the decision-maker prefers that the consistent threshold should be "very good", i.e., $\delta=s_{3}$. If we let $\lambda=0.1$, then a new LHPR $P^{(1)}$ can be derived by applying Step 3 of Algorithm 5:

$$
P^{(1)}=\left(\begin{array}{ccc}
\left\langle h_{2}, s_{0}\right\rangle & \left\langle h_{2}, s_{-2.02}\right\rangle & \left\langle h_{2}, s_{-2.88}\right\rangle \\
\left\langle h_{2}, s_{2.02}\right\rangle & \left\langle h_{1}, s_{0}\right\rangle & \left\langle h_{1}, s_{1.84}\right\rangle \\
\left\langle h_{2}, s_{2.88}\right\rangle & \left\langle h_{1}, s_{-1.84}\right\rangle & \left\langle h_{1}, s_{0}\right\rangle \\
\left\langle h_{2}, s_{0.96}\right\rangle & \left\langle h_{1}, s_{-1.06}\right\rangle & \left\langle h_{1}, s_{-2.82}\right\rangle \\
\left\langle h_{2}, s_{-1.86}\right\rangle & \left\langle h_{2}, s_{-2.08}\right\rangle & \left\langle h_{2}, s_{-1.14}\right\rangle \\
\left\langle h_{2}, s_{-0.96}\right\rangle & \left\langle h_{2}, s_{1.86}\right\rangle \\
\left\langle h_{1}, s_{1.06}\right\rangle & \left\langle h_{2}, s_{2.08}\right\rangle \\
\left\langle h_{1}, s_{2.82}\right\rangle & \left\langle h_{2}, s_{1.14}\right\rangle \\
\left\langle h_{1}, s_{0}\right\rangle & \left\langle h_{2}, s_{1.92}\right\rangle \\
\left\langle h_{2}, s_{-1.92}\right\rangle & \left\langle h_{2}, s_{0}\right\rangle
\end{array}\right)
$$

and $D\left(P^{(1)}\right)=\left\langle h_{2}, s_{3.08}\right\rangle \succ s_{3}$, thus the algorithm is terminated. Furthermore, if $\delta$ is fixed by "very good" $\left(s_{3}\right)$, "middle of very good and extremely good $\left(s_{3.5}\right)$ ", and "extremely good" $\left(s_{4}\right)$, respectively, the numbers of iterations with respect to the values of $\lambda$ have been illustrated in Fig. 5. Note that we use $s_{3.9999}$ to approximate the case $\delta=s_{4}$.

Besides, we conduct another simulation to explore the influence of the parameters. Given $S$ and $\bar{H}$ in Example 1, we ran Algorithm 5 and compute $m$ by randomly generating reciprocal LHPRs. Especially, we test $n=2,3, \ldots, 9$ and $\lambda=0.1,0.2, \ldots, 0.9$ while fixing $\delta=s_{3}$. By repeating the procedures 1000 times, the averaging values of of $m$ are derived and shown in Fig. 6. Basically, the bigger $n$ results in the bigger $m$. But the influence of $n$ is less than those of $\lambda$ and $\delta$, according to Figs. 5-6.

Similar to the existing studies, the threshold $\delta$ is determined by a decision-maker. However, the use of linguistic threshold makes its determination much easier. As can be seen in Example 6, the threshold is labeled by interpretable meaning naturally. Moreover, based on the virtual linguistic model, the decision-maker could express the threshold within a finer granularity of the given LTS, like $s_{3.5}$ in Example 6. 


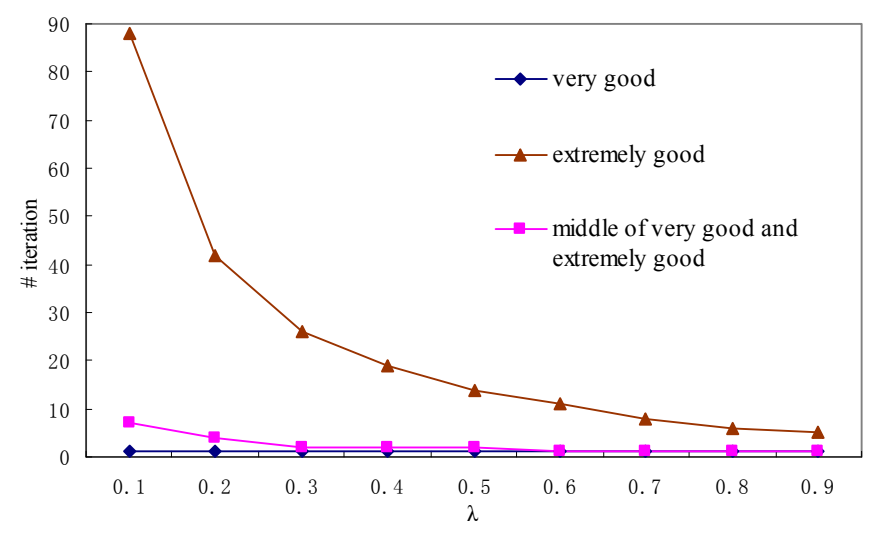

Fig. 5. Numbers of iterations with respect to $\lambda$ based on the LHPR in Example 2

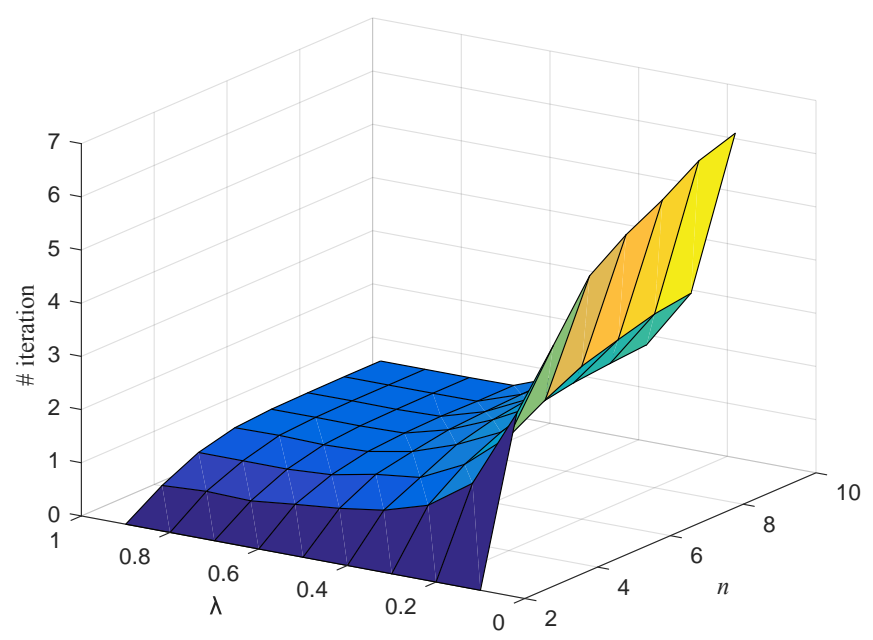

Fig. 6. Numbers of iterations with respect to $n$ and $\lambda$ based on randomly generated reciprocal LHPRs

\section{EXPERIMENTS AND DISCUSSIONS}

\section{A. An application of audits on natural resources and assets}

The Chinese government will audit all the main leaders of the Party and local governments with respect to the natural resources and assets when they are outgoing, in order to supervise their work of fulfilling the economic and environmental responsibility. Currently, Chinese scholars and auditors are seeking for proper methodologies to meet the requirement of outgoing audits. Generally, the evaluation system is very complex mainly because: (1) there are many quantitative and qualitative criteria which should be evaluated; (2) the evaluation information of several criteria, such as the reasonableness of policy, can only be assessed by experts' subjective opinions. A hierarchical structure can be considered to construct the evaluation system. The following five criteria could be included in the first level of the hierarchy: (1) responsibility of policy implementation $\left(v_{1}\right),(2)$ responsibility of decision-making $\left(v_{2}\right)$, (3) responsibility of management $\left(v_{3}\right)$, (4) responsibility of supervision $\left(v_{4}\right),(5)$ responsibility of self-discipline $\left(v_{5}\right)$. In order to determine the priority of these criteria, several experts are authorized to express their subjective preferences in the framework of traditional analytical hierarchy process.

Due to the major focus of this paper, we do not aim at solving the whole problem of audit evaluation. Instead, we focus on the collection of uncertain preferences and reasonableness checking of the collected preference relation. A group of experts and auditors are authorized to express their subjective preferences by pair-wise comparisons. They agree with utilizing the following LTS for evaluations: $S=\left\{s_{-4}=\right.$ extremely less important, $s_{-3}=$ very less important, $s_{-2}=$ less important, $s_{-1}=$ slightly less important, $s_{0}=$ indifferent, $s_{1}=$ slightly important, $s_{2}=$ important, $s_{3}=$ very important, $s_{4}=$ extremely important $\}$. If they are not sufficiently confident to use a certain term, then the WHS in Example 1 can be considered. Firstly, they provided the following preferences:
$v_{1}$ is slightly important than $v_{2}$;
$v_{2}$ is less important than $v_{3}$;
$v_{3}$ is roughly important than $v_{4}$;
$v_{4}$ is more or less indifferent to $v_{5}$.

To facilitate completing the preference, Algorithm 2 is employed to provide an estimation of the remaining entries. The algorithm outputs the following LHPR:

$P=\left(\begin{array}{ccccc}\left\langle h_{0}, s_{0}\right\rangle & \left\langle h_{0}, s_{1}\right\rangle & \left\langle h_{0}, s_{-1}\right\rangle & \left\langle h_{2}, s_{1}\right\rangle & \left\langle h_{2}, s_{1}\right\rangle \\ \left\langle h_{0}, s_{-1}\right\rangle & \left\langle h_{0}, s_{0}\right\rangle & \left\langle h_{0}, s_{-2}\right\rangle & \left\langle h_{2}, s_{0}\right\rangle & \left\langle h_{2}, s_{0}\right\rangle \\ \left\langle h_{0}, s_{1}\right\rangle & \left\langle h_{0}, s_{2}\right\rangle & \left\langle h_{0}, s_{0}\right\rangle & \left\langle h_{2}, s_{2}\right\rangle & \left\langle h_{2}, s_{2}\right\rangle \\ \left\langle h_{2}, s_{-1}\right\rangle & \left\langle h_{2}, s_{0}\right\rangle & \left\langle h_{2}, s_{-2}\right\rangle & \left\langle h_{0}, s_{0}\right\rangle & \left\langle h_{1}, s_{0}\right\rangle \\ \left\langle h_{2}, s_{-1}\right\rangle & \left\langle h_{2}, s_{0}\right\rangle & \left\langle h_{2}, s_{-2}\right\rangle & \left\langle h_{1}, s_{0}\right\rangle & \left\langle h_{0}, s_{0}\right\rangle\end{array}\right)$

The LHPR is reciprocal and additively consistent. However, it may not coincide with the group's real opinion. In order to mine more accurate information, the LHPR is fed back to the group. After discussion, they revise three entries of the upper triangular matrix:

$$
l_{14}=\left\langle h_{1}, s_{2}\right\rangle, l_{25}=\left\langle h_{0}, s_{1}\right\rangle, l_{35}=\left\langle h_{1}, s_{-3}\right\rangle .
$$

The entries of the lower triangular matrix are revised accordingly. Based on Algorithm 1, the revised version is not of weak consistency. Thus Algorithm 4 is used at first to ensure the LHPR is logically right. After 16 loops, the derived weakly consistent LHPR is as follows:

$$
P^{(16)}=\left(\begin{array}{ccc}
\left\langle h_{2}, s_{0}\right\rangle & \left\langle h_{2}, s_{1}\right\rangle & \left\langle h_{2}, s_{0.02}\right\rangle \\
\left\langle h_{2}, s_{-1}\right\rangle & \left\langle h_{2}, s_{0}\right\rangle & \left\langle h_{2}, s_{-0.98}\right\rangle \\
\left\langle h_{2}, s_{-0.02}\right\rangle & \left\langle h_{2}, s_{0.98}\right\rangle & \left\langle h_{2}, s_{0}\right\rangle \\
\left\langle h_{2}, s_{-1.49}\right\rangle & \left\langle h_{2}, s_{-0.34}\right\rangle & \left\langle h_{2}, s_{-1.32}\right\rangle \\
\left\langle h_{2}, s_{-0.49}\right\rangle & \left\langle h_{2}, s_{0.36}\right\rangle & \left\langle h_{2}, s_{0.28}\right\rangle \\
\left\langle h_{2}, s_{1.49}\right\rangle & \left\langle h_{2}, s_{0.49}\right\rangle \\
\left\langle h_{2}, s_{0.34}\right\rangle & \left\langle h_{2}, s_{-0.36}\right\rangle \\
\left\langle h_{2}, s_{1.32}\right\rangle & \left\langle h_{2}, s_{-0.28}\right\rangle \\
\left\langle h_{2}, s_{0}\right\rangle & \left\langle h_{2}, s_{-0.85}\right\rangle \\
\left\langle h_{2}, s_{0.85}\right\rangle & \left\langle h_{2}, s_{0}\right\rangle
\end{array}\right)
$$

The degree of additive consistency of $P^{(16)}$ is $\left\langle h_{2}, s_{3.84}\right\rangle$. Meanwhile, the decision-maker requires that the threshold of satisfactory consistency is $s_{3}$. Because $\left\langle h_{2}, s_{3.84}\right\rangle \succ s_{3}$, the consistency degree of $P^{(16)}$ is satisfactory. 


\section{B. Discussions}

We have illustrated the proposed definitions and algorithms by means of some examples and a real case. In this section, we will discuss the inherent idea by comparing with some similar types of preference relations based on natural linguistic expressions, i.e., LPRs, ULPRs, and HFLPRs. Some of the major differences are listed in Table I.

The entries of these types of preference relations are fundamentally different due to the inherent uncertainties implied by their focused types of linguistic expressions. Based on specific linguistic representational models, the uncertainty of an entry of LPRs is implied by the linguistic terms, specified by their semantics. In this sense, experts have to be confident enough to express their preferences by single terms. Otherwise, preferences could be expressed by CLEs, such as ULTs, HFLTSs, LTWHs, and other artificial linguistic expression. See [35] for a detailed review. For ULPRs and HFLPRs, the uncertainty of preferences expressed by CLEs are modelled by the sets of all possible terms. From the mathematical perspective, these two techniques model the uncertainty by fixing the boundaries. However, in LHPRs, the linguistic term in a LTWH indicates the term that is the most possibly real value. And the hedge qualifies the degree of uncertainty. This is another natural way of humans' linguistic convention. Therefore, the development of LHPRs extends the way of expressing preferences in qualitative setting.

It is quite natural to visualize preference relations by means of graphs. For weak consistency, it is sufficient to consider the presence and absence of an arc. However, the weights of arcs should be taken into account when measuring additive consistency. The P-graph of a LPR is a simple graph and thus the weight of an arc can be defined by the index of the corresponding entry, as can be seen in [36], [37]. But this is not the case in HFLPRs where the P-graph is generally a multiple graph. The weight of each arc of a HFLPR can be defined by the index of each possible term in a HFLTS. See [22] for example. Different from HFLTSs, LTWHs imply multiple terms in an indirect way. The uncertainty included in hedges cannot be represented by simply listing possible terms. Therefore, fuzzy weighted graphs are introduced to visualize LHPRs. In such a manner, the P-graph of a LHPR is also a simple graph whose weights are fuzzy numbers. The same idea could be considered for the visualization of ULPRs.

Generally, satisfactory consistencies are defined by the degree of a preference relation satisfying additive consistencies. It is inevitable that the threshold depends on the risk attitude of a decision-maker and thus should be determined by the decision-maker. To ease the determination, it would be significative and helpful if the decision-maker could understand the defined measure of the degree of additive consistency directly [38]. However, most of the existing contributions, such as [22], [39], [40], utilize a real number in $[0,1]$ to serve as the threshold. No guideline has been given to select the value. The selected number is not interpretable. Thus this kind of measure remains an obstacle to use satisfactory consistencies in potential applications. Dong et al. [37] firstly tried to define this measure by means of linguistic terms.
In order that, a specific LTS with seven terms is defined to evaluate the degree of additive consistency. Based on a context-free grammar, this paper suggests the use of some LTWHs in $\mathcal{L}$. The proposed procedure is much easier than that of [37]. These two proposals improve the interpretability of consistency thresholds. But it should be noticed that, in order to guarantee the interpretability, the decision-maker has to understand the semantics of the involved linguistic terms, and new consistency improving algorithm (such as Algorithm 5) has to be designed accordingly. Thus the process is more complicated than the traditional ones with numerical thresholds.

It is interesting to note that the parameter $\lambda$ could also be linguistic values. Roughly, $\lambda$ can be interpreted in some way, such as the percentages of original information that should be considered. However, the decision-maker might be not so confident to distinguish two different values, especially when the two values are very close. In this sense, one could assign linguistic values to $\lambda$ so that the decision-maker could easily understand the selected value. If so, some preliminaries should be prepared to extend Algorithm 5 .

The proposed consistency measures and algorithms could be employed to complete unknown entries in LHPRs. As shown in Example 4 and Section VI-A, unknown entries can be estimated automatically based on the additive consistency. In fact, given a $n \times n$ LHPR, if more than $n-1$ entries are known, then some elaborate algorithms could be developed to estimate unknown entries according to the criterion of reaching the highest degree of additive consistency. Some existing strategy of traditional LPRs can be found in [41]. Moreover, the weak consistency could be used to search possible values of each unknown entries and to design interactive algorithms based on the idea in [32].

\section{CONCLUSIONS}

The model of LTWHs presents a new tool to compute with a natural type of CLEs in QDM. Based on the existing representational model, this paper has developed a new computational model including order relations and basic operations. To facilitate this type of CLEs in expressing preferences, the concept of LHPRs has been defined. More importantly, several consistency measures have been proposed and their properties have been discovered so that the quality of a given LHPR can be identified. The consistency measures have been defined by specific transitivity and illustrated by fuzzy weighted digraphs. The main contributions of this paper are as follows:

(1) The theoretical foundation of applying LHPRs in the framework of QDM with preference relation has been established. We have introduced the definition of LHPRs and their consistency measures. Especially, we have focused on the properties of weak consistency and additive consistency.

(2) The approaches for checking the weak consistency and additive consistency of a LHPR have been proposed. The algorithms have been developed based on the definitions and are easy to implement. The effectiveness and convergence of the algorithms have also been proven.

(3) The automatic algorithms for consistency improving have been developed for the cases when a LHPR is not weakly 
TABLE I

CHARACTERISTICS OF THE COMPARATIVE TECHNIQUES

\begin{tabular}{lllll}
\hline Technique & Underlying linguistic model & Uncertainty representation & Consistency visualization & Satisfactory consistency measure \\
\hline LPR & Any & Semantics of a term & Simple graph & Numbers in $[0,1]$ or linguistic terms \\
ULPR & ULT & Linguistic interval & N/A & Numbers in $[0,1]$ \\
HFLPR & HFLTS & A set of terms & Multiple graph & Numbers in $[0,1]$ \\
LHPR & LTWH & Weakened hedge & Fuzzy weighted graph & linguistic terms \\
\hline
\end{tabular}

consistent, or when the degree of additive consistency is not satisfied by a decision-maker.

Meanwhile, this proposal possesses some limitations as well. These results in the following open problems:

(1) Techniques for exploiting priorities implied by the LHPR should be developed. This paper presents approaches to ensure that a LHPR is meaningful. To enable the use of LHPRs in real applications, algorithms should be developed to either rank the objects or obtain the relative weights of the objects. Roughly, semantics-based strategy and eigenvector-based strategy are both possible solutions.

(2) Techniques for group decision making with LHPRs are required. In this case, two vital points, i.e., the synthesis of individual LHPRs and the group consensus, should be addressed at first. The techniques in (1) are also necessary. Specifically, the consensus could be measured by linguistic thresholds, similar to the idea of this paper. And the synthesis could be achieved by maximum the group's consensus.

(3) The multiplicative consistency of LHPRs should be further studied. Multiplicative transitivity is more useful than additive consistency in some sense [26]. However, it is not straightforward to study the multiplicative transitivity by graphs. Thus it is not the main focus of this paper. The multiplicative consistency could be checked and improved by extending the idea in [26].

(4) LHPRs based on unbalanced LTSs are also interesting. Based on the representational model, LTWHs in a LHPR could be a special case of unbalanced LTSs where the semantics of terms should be defined by triangular fuzzy numbers. For more general cases, LHPRs might be also useful. As possible solutions, the model of LTWHs could be reconstructed based on the ordered structured model, or an uniformly distributed LTS could be employed for transformation, like the idea in [42].

\section{REFERENCES}

[1] D. Dubois, H. Fargier, and P. Perny, "Qualitative decision theory with preference relations and comparative uncertainty: An axiomatic approach," Artif. Intell., vol. 148, no. 1, pp. 219-260, Aug. 2003.

[2] L. A. Zadeh, "The concept of a linguistic variable and its application to approximate reasoning-I,' Inf. Sci., vol. 8, no. 3, pp. 199-249, 1975.

[3] R. Degani and G. Bortolan, "The problem of linguistic approximation in clinical decision making," Int. J. Approx. Reason., vol. 2, no. 2, pp. 143-162, 1988.

[4] R. R. Yager, "A new methodology for ordinal multiple aspect decisions based on fuzzy sets," Decis. Sci., vol. 12, pp. 589-600, 1981.

[5] F. Herrera, E. Herrera-Viedma, and J. Verdegay, "Direct approach processes in group decision making using linguistic OWA operators," Fuzzy Sets Syst., vol. 79, pp. 175-190, 1996.

[6] F. Herrera and L. Martínez, "A 2-tuple fuzzy linguistic representation model for computing with words," IEEE Trans. Fuzzy Syst., vol. 8, no. 6, pp. 746-752, Dec. 2000.
[7] Z. S. Xu, "A method based on linguistic aggregation operators for group decision making with linguistic preference relations," Inf. Sci., vol. 166, no. 1, pp. 19-30, Oct. 2004.

[8] Z. S. Xu and H. Wang, "On the syntax and semantics of virtual linguistic terms for inf. fusion in decision making," Inf. Fusion, vol. 34, pp. $43-$ 48, Mar. 2017.

[9] F. J. Cabrerizo, R. Al-Hmouz, A. Morfeq, A. S. Balamash, M. A. Martínez, and E. Herrera-Viedma, "Soft consensus measures in group decision making using unbalanced fuzzy linguistic information," Soft Comput., vol. 21, no. 11, pp. 3037-3050, Jun 2017. [Online]. Available: https://doi.org/10.1007/s00500-015-1989-6

[10] J. A. Morente-Molinera, J. Mezei, C. Carlsson, and E. Herrera-Viedma "Improving supervised learning classification methods using multigranular linguistic modeling and fuzzy entropy," IEEE Trans. Fuzzy Syst., vol. 25 , no. 5 , pp. 1078-1089, Oct 2017.

[11] Z. S. Xu, "Uncertain linguistic aggregation operators based approach to multiple attribute group decision making under uncertain linguistic environment," Inf. Sci., vol. 168, no. 1, pp. 171-184, Dec. 2004.

[12] R. M. Rodríguez, L. Martínez, and F. Herrera, "Hesitant fuzzy linguistic term sets for decision making," IEEE Trans. Fuzzy Syst., vol. 20, no. 1, pp. 109-119, Feb. 2012.

[13] H. C. Liao, Z. S. Xu, E. Herrera-Viedma, and F. Herrera, "Hesitant fuzzy linguistic term set and its application in decision making: A state-of-the-art survey," Int. J. Fuzzy Syst., Dec 2017. [Online]. Available: https://doi.org/10.1007/s40815-017-0432-9

[14] Y. Tang and J. Zheng, "Linguistic modelling based on semantic similarity relation among linguistic labels," Fuzzy Sets Syst., vol. 157, no. 12, pp. 1662-1673, Jun. 2006.

[15] H. Wang, Z. S. Xu, and X.-J. Zeng, "Linguistic terms with weakened hedges: A model for qualitative decision making under uncertainty," Inf. Sci., vol. 433-434, no. 3, pp. 37-54, Apr. 2018.

[16] T. L. Saaty, "A scaling method for priorities in hierarchical structures," J. Math. Psychol., vol. 15, no. 3, pp. 234-281, Jun. 1977.

[17] S. Orlovsky, "Decision-making with a fuzzy preference relation," Fuzzy Sets Syst., vol. 1, no. 3, pp. 155-167, Jul. 1978.

[18] F. Herrera and E. Herrera-Viedma, "Choice functions and mechanisms for linguistic preference relations," Eur. J. Oper. Res., vol. 120, no. 1, pp. 144-161, Jan. 2000.

[19] Z. S. Xu, "Deviation measures of linguistic preference relations in group decision making," Omega, vol. 33, no. 3, pp. 249-254, Jun. 2005.

[20] Z. S. Xu, "A direct approach to group decision making with uncertain additive linguistic preference relations," Fuzzy Opti. Decis. Ma., vol. 5, no. 1, pp. 21-32, 2006.

[21] B. Zhu and Z. S. Xu, "Consistency measures for hesitant fuzzy linguistic preference relations," IEEE Trans. Fuzzy Syst., vol. 22, no. 1, pp. 35-45, Feb. 2014.

[22] H. Wang and Z. S. Xu, "Some consistency measures of extended hesitant fuzzy linguistic preference relations," Inf. Sci., vol. 297, pp. 316-331, Mar. 2015.

[23] E. Herrera-Viedma, F. Herrera, F. Chiclana, and M. Luque, "Some issues on consistency of fuzzy preference relations," Eur. J. Oper. Res., vol. 154, no. 1, pp. 98-109, Apr. 2004.

[24] T. Tanino, "Fuzzy preference orderings in group decision making," Fuzzy Sets Syst., vol. 12, no. 2, pp. 117-131, Feb. 1984.

[25] T. Tanino, "Fuzzy preference relations in group decision making," in Non-conventional preference relations in decision making. Springer, 1988, pp. 54-71.

[26] F. Chiclana, E. Herrera-Viedma, S. Alonso, and F. Herrera, "Cardinal consistency of reciprocal preference relations: A characterization of multiplicative transitivity," IEEE Trans. Fuzzy Syst., vol. 17, no. 1, pp. 14-23, Feb 2009.

[27] Z. B. Wu and J. P. Xu, "A consistency and consensus based decision support model for group decision making with multiplicative preference relations," Decis. Support Syst., vol. 52, no. 3, pp. 757-767, Feb. 2012.

[28] Y. J. Xu, R. Patnayakuni, and H. M. Wang, "The ordinal consistency of a fuzzy preference relation," Inf. Sci., vol. 224, pp. 152-164, Mar. 2013. 
[29] M. De Cock and E. E. Kerre, "Fuzzy modifiers based on fuzzy relations," Inf. Sci., vol. 160, no. 1, pp. 173-199, Mar. 2004

[30] J. Lawry, "A framework for linguistic modelling," Artif. Intell., vol. 155, no. 1-2, pp. 1-39, May 2004.

[31] K.-C. Lin and M.-S. Chern, "The fuzzy shortest path problem and its most vital arcs," Fuzzy Sets Syst., vol. 58, no. 3, pp. 343-353, Sep. 1993.

[32] H. Wang and Z. S. Xu, "Interactive algorithms for improving incomplete linguistic preference relations based on consistency measures," Appl. Soft Comput., vol. 42, pp. 66-79, May 2016.

[33] J. Ma, Z. P. Fan, Y. P. Jiang, J. Y. Mao, and L. Ma, "A method for repairing the inconsistency of fuzzy preference relations," Fuzzy Sets Syst., vol. 157, no. 1, pp. 20-33, Jan. 2006.

[34] T. L. Saaty and M. S. Ozdemir, "Why the magic number seven plus or minus two," Math. Comput. Model., vol. 38, no. 3-4, pp. 233-244, Aug. 2003.

[35] H. Wang, Z. S. Xu, and X.-J. Zeng, "Modeling complex linguistic expressions in qualitative decision making: An overview," Knowl. Based Syst., 2018.

[36] J. L. Garca-Lapresta and L. C. Meneses, "Modeling rationality in a linguistic framework," Fuzzy Sets Syst., vol. 160, no. 22, pp. 3211-3223, 2009.

[37] Y. C. Dong, W. C. Hong, and Y. F. Xu, "Measuring consistency of linguistic preference relations: a 2-tuple linguistic approach," Soft Comput., vol. 17, no. 11, pp. 2117-2130, 2013.

[38] F. Herrera, S. Alonso, F. Chiclana, and E. Herrera-Viedma, "Computing with words in decision making: foundations, trends and prospects," Fuzzy Opti. Decis. Ma., vol. 8, no. 4, pp. 337-364, 2009.

[39] H. Y. Chen, L. G. Zhou, and B. Han, "On compatibility of uncertain additive linguistic preference relations and its application in the group decision making," Knowl. Based Syst., vol. 24, no. 6, pp. 816-823, 2011.

[40] Y. C. Dong, W. C. Hong, Y. F. Xu, and S. Yu, "Selecting the individual numerical scale and prioritization method in the analytic hierarchy process: A 2-tuple fuzzy linguistic approach," IEEE Trans. Fuzzy Syst., vol. 19, no. 1, pp. 13-25, 2011.

[41] M. Ureña, F. Chiclana, J. Morente-Molinera, and E. Herrera-Viedma, "Managing incomplete preference relations in decision making: A review and future trends," Inf. Sci., vol. 302, no. 1, pp. 14-32, Jan. 2015.

[42] F. Herrera, E. Herrera-Viedma, and L. Martínez, "A fusion approach for managing multi-granularity linguistic term sets in decision making," Fuzzy Sets Syst., vol. 114, no. 1, pp. 43-58, Aug. 2000. 